\title{
LNCaP Atlas: Gene expression associated with in vivo progression to castration-recurrent prostate cancer
}

\author{
Tammy L Romanuik, Gang Wang, Olena Morozova, Allen Delaney, Marco A Marra, Marianne D Sadar
}

\begin{abstract}
Background: There is no cure for castration-recurrent prostate cancer (CRPC) and the mechanisms underlying this stage of the disease are unknown.

Methods: We analyzed the transcriptome of human LNCaP prostate cancer cells as they progress to CRPC in vivo using replicate LongSAGE libraries. We refer to these libraries as the LNCaP atlas and compared these gene expression profiles with current suggested models of CRPC.

Results: Three million tags were sequenced using in vivo samples at various stages of hormonal progression to reveal 96 novel genes differentially expressed in CRPC. Thirty-one genes encode proteins that are either secreted or are located at the plasma membrane, 21 genes changed levels of expression in response to androgen, and 8 genes have enriched expression in the prostate. Expression of 26, 6, 12, and 15 genes have previously been linked to prostate cancer, Gleason grade, progression, and metastasis, respectively. Expression profiles of genes in CRPC support a role for the transcriptional activity of the androgen receptor (CCNH, CUEDC2, FLNA, PSMA7), steroid synthesis and metabolism (DHCR24, DHRS7, ELOVL5, HSD17B4, OPRK1), neuroendocrine (ENO2, MAOA, OPRK1, S100A10, TRPM8), and proliferation (GAS5, GNB2L1, MT-ND3, NKX3-1, PCGEM1, PTGFR, STEAP1, TMEM3OA), but neither supported nor discounted a role for cell survival genes.
\end{abstract}

Conclusions: The in vivo gene expression atlas for LNCaP was sequenced and support a role for the androgen receptor in CRPC.

\section{Background}

Systemic androgen-deprivation therapy by orchiectomy or agonists of gonadotropic releasing hormone are routinely used to treat men with metastatic prostate cancer to reduce tumor burden and pain. This therapy is based on the dependency of prostate cells for androgens to grow and survive. The inability of androgen-deprivation therapy to completely and effectively eliminate all metastatic prostate cancer cell populations is manifested by a predictable and inevitable relapse, referred to as castration-recurrent prostate cancer (CRPC). CRPC is the end stage of the disease and fatal to the patient within 16-18 months of onset.

\footnotetext{
* Correspondence: msadar@bcgsc.ca

Genome Sciences Centre, British Columbia Cancer Agency, Vancouver, British Columbia, Canada
}

(c) 2010 Romanuik et al; licensee BioMed Central Ltd. This is an Open Access article distributed under the terms of the Creative Commons Attribution License (http://creativecommons.org/licenses/by/2.0), which permits unrestricted use, distribution, and reproduction in any medium, provided the original work is properly cited.
The mechanisms underlying progression to CRPC are unknown. However, there are several models to explain its development. One such model indicates the involvement of the androgen signaling pathway [1-4]. Key to this pathway is the androgen receptor (AR) which is a steroid hormone receptor and transcription factor. Mechanisms of progression to CRPC that involve or utilize the androgen signaling pathway include: hypersensitivity due to $A R$ gene amplification [5,6]; changes in AR co-regulators such as nuclear receptor coactivators (NCOA1 and NCOA2) [7,8]; intraprostatic de novo synthesis of androgen [9] or metabolism of AR ligands from residual adrenal androgens [10,11]; AR promiscuity of ligand specificity due to mutations [12]; and ligandindependent activation of AR by growth factors [protein kinase A (PKA), interleukin 6 (IL6), and epidermal growth factor (EGF)] [13-15]. Activation of the AR can be determined by assaying for the expression of target 
genes such as prostate-specific antigen (PSA) [16]. Other models of CRPC include the neuroendocrine differentiation [17], the stem cell model [18] and the imbalance between cell growth and cell death [3]. It is conceivable that these models may not mutual exclusive. For example altered AR activity may impact cell survival and proliferation.

Here, we describe long serial analysis of gene expression (LongSAGE) libraries $[19,20]$ made from RNA sampled from biological replicates of the in vivo LNCaP Hollow Fiber model of prostate cancer as it progresses to the castration-recurrent stage. Gene expression signatures that were consistent among the replicate libraries were applied to the current models of CRPC.

\section{Methods}

\section{In vivo $\mathrm{LNCaP}$ Hollow Fiber model}

The LNCaP Hollow Fiber model of prostate cancer was performed as described previously [21-23]. All animal experiments were performed according to a protocol approved by the Committee on Animal Care of the University of British Columbia. Serum PSA levels were determined by enzymatic immunoassay kit (Abbott Laboratories, Abbott Park, IL, USA). Fibers were removed on three separate occasions representing different stages of hormonal progression that were androgen-sensitive (AS), responsive to androgen-deprivation (RAD), and castration-recurrent (CR). Samples were retrieved immediately prior to castration (AS), as well as 10 (RAD) and 72 days $(\mathrm{CR})$ post-surgical castration.

\section{RNA sample generation, processing, and quality control}

Total RNA was isolated immediately from cells harvested from the in vivo Hollow Fiber model using TRIZOL Reagent (Invitrogen) following the manufacturer's instructions. Genomic DNA was removed from RNA samples with DNaseI (Invitrogen). RNA quality and quantity were assessed by the Agilent 2100 Bioanalyzer (Agilent Technologies, Mississauga, ON, Canada) and RNA 6000 Nano LabChip kit (Caliper Technologies, Hopkinton, MA, USA).

\section{Quantitative real-time polymerase chain reaction}

Oligo-d(T)-primed total RNAs $(0.5 \mu$ g per sample) were reverse-transcribed with SuperScript III (Invitrogen Life Technologies, Carlsbad, CA, USA). An appropriate dilution of cDNA and gene-specific primers were combined with SYBR Green Supermix (Invitrogen) and amplified in ABI 7900 real-time PCR machine (Applied Biosystems, Foster City, CA, USA). All qPCR reactions were performed in triplicate. The threshold cycle number $(\mathrm{Ct})$ and expression values with standard deviations were calculated in Excel. Primer sequences for real-time PCRs are: KLK3, F': 5'-CCAAGTTCATGCTGTGTGCT-3' and
R:' 5'-CCCATGACGTGATACCTTGA-3'; glyceraldehyde3-phosphate (GAPDH), F': 5'-CTGACTTCAACAGCGACACC-3' and R:' 5'-TGCTGTAGCCAAATTCGTTG-3'). Real-time amplification was performed with initial denaturation at $95^{\circ} \mathrm{C}$ for $2 \mathrm{~min}$, followed by 40 cycles of twostep amplification $\left(95^{\circ} \mathrm{C}\right.$ for $15 \mathrm{sec}, 55^{\circ} \mathrm{C}$ for $\left.30 \mathrm{sec}\right)$.

\section{LongSAGE library production and sequencing}

RNA from the hollow fibers of three mice (biological replicates) representing different stages of prostate cancer progression (AS, RAD, and CR) were used to make a total of nine LongSAGE libraries. LongSAGE libraries were constructed and sequenced at the Genome Sciences Centre, British Columbia Cancer Agency. Five micrograms of starting total RNA was used in conjunction with the Invitrogen I-SAGE Long kit and protocol with alterations [24]. Raw LongSAGE data are available at Gene Expression Omnibus [25] as series accession number GSE18402. Individual sample accession numbers are as follows: S1885, GSM458902; S1886, GSM458903; S1887, GSM458904; S1888, GSM458905; S1889, GSM458906; S1890, GSM458907; S1891, GSM458908; S1892, GSM458909; and S1893, GSM458910.

\section{Gene expression analysis}

LongSAGE expression data was analyzed with DiscoverySpace 4.01 software [26]. Sequence data were filtered for bad tags (tags with one N-base call) and linker-derived tags (artifact tags). Only LongSAGE tags with a sequence quality factor (QF) greater than 95\% were included in analysis. The phylogenetic tree was constructed with a distance metric of 1-r (where "r" equals the Pearson correlation coefficient). Correlations were computed (including tag counts of zero) using the Regress program of the Stat package written by Ron Perlman, and the tree was optimized using the Fitch program [27] in the Phylip package [28]. Graphics were produced from the tree files using the program TreeView [29]. Tag clustering analysis was performed using the Poisson distribution-based K-means clustering algorithm. The K-means algorithm clusters tags based on count into ' $K$ ' partitions, with the minimum intracluster variance. PoissonC was developed specifically for the analysis of SAGE data [30]. The java implementation of the algorithm was kindly provided by Dr. Li Cai (Rutgers University, NJ, USA). An optimal value for $\mathrm{K}$ $(\mathrm{K}=10)$ was determined [31].

\section{Principle component analysis}

Principle component analysis was performed using GeneSpring $^{\mathrm{mm}}$ software version 7.2 (Silicon Genetics, CA). Affymetrix datasets of clinical prostate cancer and normal tissue were downloaded from Gene Expression Omnibus [25] (accession numbers: GDS1439 and 
GDS1390) and analyzed in GeneSpring ${ }^{\text {max }}$. Of the 96 novel CR-associated genes, 76 genes had corresponding Affymetrix probe sets. These probe sets were applied as the gene signature in this analysis. Principle component (PC) scores were calculated according to the standard correlation between each condition vector and each principle component vector.

\section{Results}

\section{LongSAGE library and tag clustering}

RNA isolated from the LNCaP Hollow Fiber model was obtained from at least three different mice $(13 \mathrm{~N}, 15 \mathrm{~N}$, and 13R; biological replicates) at three stages of cancer progression that were androgen-sensitive (AS), responsive to androgen-deprivation (RAD), and castrationrecurrent (CR). To confirm that the samples represented unique disease-states, we determined the levels of $K L K 3$ mRNA, a biomarker that correlates with progression, using quantitative real time-polymerase chain reaction (qRT-PCR). As expected, KLK3 mRNA levels dropped in the stage of cancer progression that was RAD versus AS (58\%, 49\%, and 37\%), and rose in the stage of cancer progression that was CR versus RAD $(229 \%, 349 \%$, and $264 \%$ ) for mice $13 \mathrm{R}, 15 \mathrm{~N}$, and $13 \mathrm{~N}$, respectively (Additional file 1). Therefore, we constructed nine LongSAGE libraries, one for each stage and replicate.

LongSAGE libraries were sequenced to 310,072 339,864 tags each, with a combined total of $2,931,124$ tags, and filtered to leave only useful tags for analysis (Table 1). First, bad tags were removed because they contain at least one N-base call in the LongSAGE tag sequence. The sequencing of the LongSAGE libraries was base called using PHRED software. Tag sequencequality factor (QF) and probability was calculated to ascertain which tags contain erroneous base-calls. The second line of filtering removed LongSAGE tags with probabilities less than 0.95 (QF $<95 \%)$. Linkers were introduced into SAGE libraries as known sequences utilized to amplify ditags prior to concatenation. At a low frequency, linkers ligate to themselves creating linkerderived tags (LDTs). These LDTs do not represent transcripts and were removed from the LongSAGE libraries. A total of 2,305,589 useful tags represented by 263,197 tag types remained after filtering. Data analysis was carried out on this filtered data.

The LongSAGE libraries were hierarchically clustered and displayed as a phylogenetic tree. In most cases, LongSAGE libraries made from the same disease stage (AS, RAD, or CR) clustered together more closely than LongSAGE libraries made from the same biological replicate (mice $13 \mathrm{~N}, 15 \mathrm{~N}$, or 13R; Figure 1). This suggests the captured transcriptomes were representative of disease stage with minimal influence from biological variation.
Identification of groups of genes that behave similarly during progression of prostate cancer was conducted through K-means clustering of tags using the PoissonC algorithm [30]. For each biological replicate (mice 13N, $15 \mathrm{~N}$, or $13 \mathrm{R}$ ), all tag types were clustered that had a combined count greater than ten in the three libraries representing disease stages (AS, RAD, and CR) and mapped unambiguously sense to a transcript in reference sequence (RefSeq; February $28^{\text {th }}, 2008$ ) [32] using DiscoverySpace4 software [33]. By plotting within cluster dispersion (i.e., intracluster variance) against a range of $\mathrm{K}$ (number of clusters; Additional file 1, Figure S2), we determined that ten clusters best embodied the expression patterns present in each biological replicate. This was decided based on the inflection point in the graph (Additional file 1, Figure S2), showing that after reaching $K=10$, increasing the number of $K$ did not substantially reduce the within cluster dispersion. $\mathrm{K}$-means clustering was performed over 100 iterations, so that tags would be placed in clusters that best represent their expression trend. The most common clusters for each tag are displayed (Figure 2). In only three instances, there were similar clusters in just two of the three biological replicates. Consequently, consistent changes in gene expression during progression were represented in 11 patterns. Differences among expression patterns for each biological replicate may be explained by biological variation, the probability of sampling a given LongSAGE tag, and/or imperfections in K-means clustering (e.g, variance may not be a good measure of cluster scatter).

\section{Gene ontology enrichment analysis}

We conducted Gene Ontology (GO) [34] enrichment analysis using Expression Analysis Systematic Explorer (EASE) [35] software to determine whether specific GO annotations were over-represented in the K-means clusters. Enrichment was defined by the EASE score (p-value s 0.05 ) generated during comparison to all the other clusters in the biological replicate. This analysis was done for each biological replicate (3 mice: $13 \mathrm{~N}, 15 \mathrm{~N}$, or 13R).

To enable visual differences between the 11 expression trends, the clusters were amalgamated into five major trends: group 1, up during progression; group 2, down during progression; group 3 , peak in the RAD stage; group 4, constant during progression; and group 5, valley in RAD stage (Figure 2). To be consistent, the GO enrichment data was combined into five major trends which resulted in redundancy in GO terms. To simplify the GO enrichment data, similar terms were pooled into representative categories. Categorical gene ontology enrichments of the five major expression trends are shown in Figure 3. These data indicate that steroid binding, heat shock protein activity, de-phosphorylation activity, and glycolysis all 
Table 1 Composition of LongSAGE libraries

\begin{tabular}{|c|c|c|c|c|c|c|c|c|c|}
\hline Library & S1885 & S1886 & S1887 & S1888 & S1889 & S1890 & S1891 & S1892 & S1893 \\
\hline Mouse-Condition & $13 \mathrm{~N}-\mathrm{AS}^{*}$ & 13N-RAD + & $13 \mathrm{~N}-\mathrm{CR} \neq$ & $15 \mathrm{~N}-\mathrm{AS}$ & $15 N-R A D$ & $15 \mathrm{~N}-\mathrm{CR}$ & 13R-AS & 13R-RAD & $13 R-C R$ \\
\hline Unfiltered Total Tags & 310,516 & 318,102 & 339,864 & 338,210 & 310,072 & 326,870 & 337,546 & 314,440 & 335,504 \\
\hline No. of Bad Tags & 955 & 1,010 & 1,083 & 1,097 & 983 & 737 & 900 & 744 & 832 \\
\hline \multicolumn{10}{|l|}{ Minus Bad Tags } \\
\hline Total Tags & 309,561 & 317,092 & 338,781 & 337,113 & 309,089 & 326,133 & 336,646 & 313,696 & 334,672 \\
\hline Tag Types & 79,201 & 96,973 & 99,730 & 81,850 & 84,499 & 88,249 & 79,859 & 91,438 & 90,675 \\
\hline No. of Duplicate Ditags & 19,761 & 12,220 & 12,678 & 21,973 & 17,471 & 12,836 & 24,552 & 12,786 & 13,127 \\
\hline$\%$ of Duplicate Ditags & 6.38 & 3.85 & 3.74 & 6.52 & 5.65 & 3.94 & 7.29 & 4.08 & 3.92 \\
\hline Average QF§ of Tags & 0.85 & 0.88 & 0.87 & 0.86 & 0.89 & 0.88 & 0.88 & 0.80 & 0.87 \\
\hline No. of Tags QF $<0.95$ & 63,057 & 62,872 & 71,576 & 68,993 & 54,627 & 54,470 & 68,981 & 101,215 & 69,647 \\
\hline \multicolumn{10}{|l|}{$Q \geq 0.95$} \\
\hline Total Tags & 246,504 & 254,220 & 267,205 & 268,120 & 254,462 & 271,663 & 267,665 & 212,481 & 265,025 \\
\hline Tag Types & 52,033 & 67,542 & 66,748 & 52,606 & 59,374 & 64,985 & 53,715 & 54,682 & 64,837 \\
\hline Total Tags Combined & & & & & $2,307,345$ & & & & \\
\hline Tag Types Combined & & & & & 263,199 & & & & \\
\hline No. of LDTs II Type I & 124 & 72 & 174 & 179 & 84 & 186 & 164 & 118 & 301 \\
\hline No. of LDTs Type II & 19 & 9 & 54 & 56 & 33 & 40 & 60 & 24 & 59 \\
\hline \multicolumn{10}{|l|}{ Minus LDTs } \\
\hline Total Tags & 246,361 & 254,139 & 266,977 & 267,885 & 254,345 & 271,437 & 267,441 & 212,339 & 264,665 \\
\hline Tag Types & 52,031 & 67,540 & 66,746 & 52,604 & 59,372 & 64,983 & 53,713 & 54,680 & 64,835 \\
\hline Total Tags Combined & & & & & $2,305,589$ & & & & \\
\hline Tag Types Combined & & & & & 263,197 & & & & \\
\hline
\end{tabular}

* AS, Androgen-sensitive/

† RAD, Responsive to androgen-deprivation.

‡ CR, Castration-recurrent.

$\S \mathrm{QF}$, Quality Factor.

II LDTs, Linker-derived tags.

decreased in the stage that was RAD, but increased again in the stage that was CR. Interestingly, steroid hormone receptor activity continues to increase throughout progression. Both of these expression trends were observed for genes with GO terms for transcription factor activity or secretion. The GO categories for genes with kinase activity and signal transduction displayed expression trends with peaks and valleys at the stage that was RAD. The levels of expression of genes involved in cell adhesion rose in the stage that was RAD, but dropped again in the stage that was CR.

Altogether, genes with functional categories that were enriched in expression trends may be consistent with the AR signaling pathway playing a role in progression of

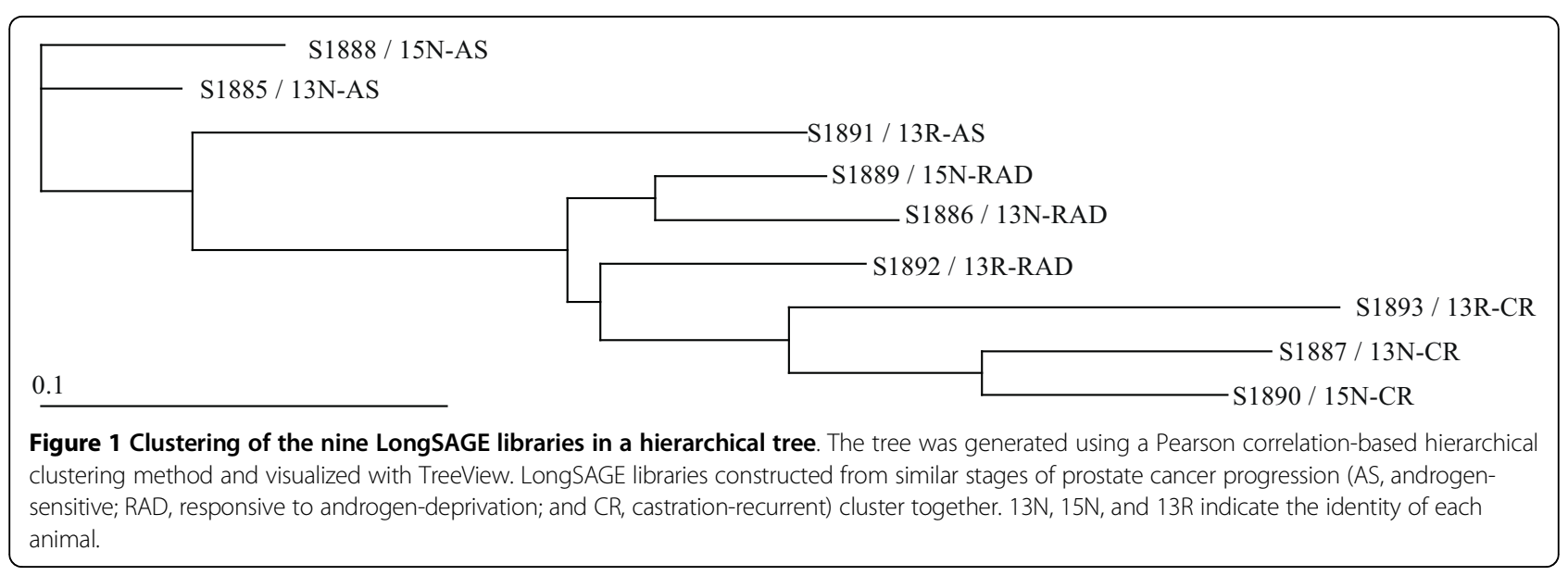




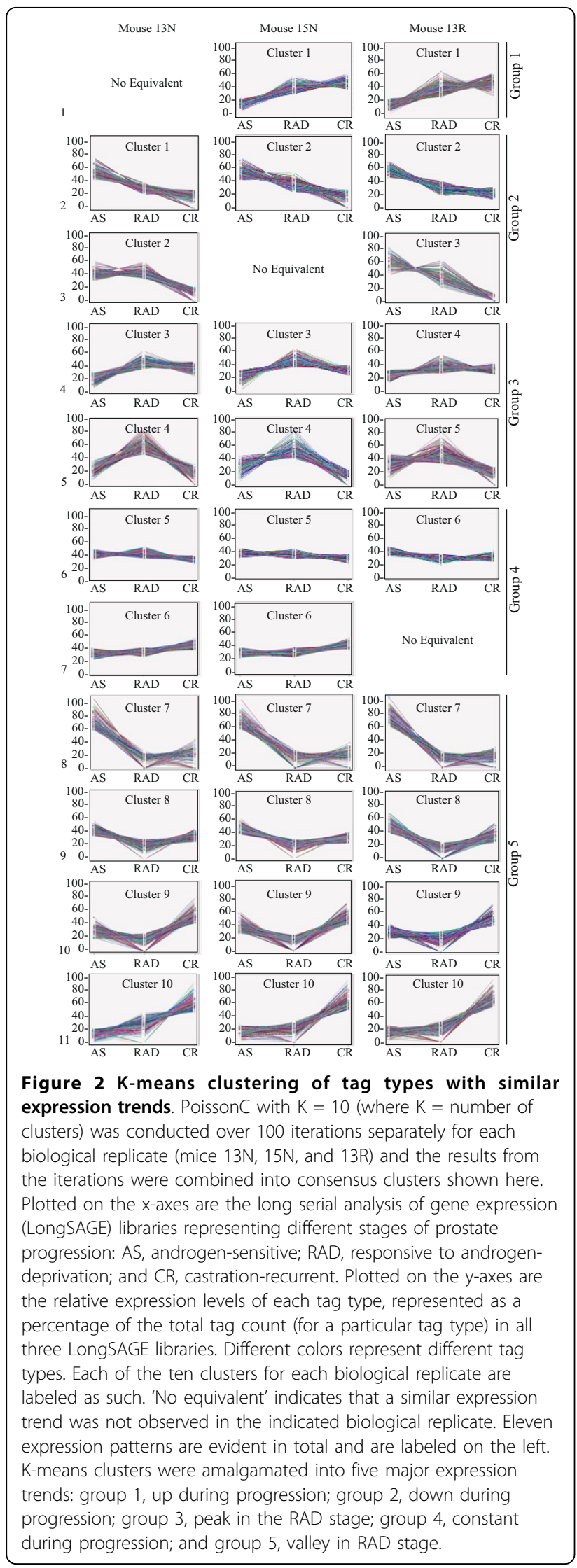

prostate cancer to castration-recurrence (Figure 3). For example, GO terms steroid binding, steroid hormone receptor activity, heat shock protein activity, chaperone activity, and kinase activity could represent the cytoplasmic events of AR signaling. GO terms transcription factor activity, regulation of transcription, transcription corepression activity, and transcription co-activator activity could represent the nuclear events of AR signaling. AR-mediated gene transcription may result in splicing and protein translation, to regulate general cellular processes such as proliferation (and related nucleotide synthesis, DNA replication, oxidative phosphorylation, oxioreductase activity, and glycolysis), secretion, and differentiation.

It should be noted, however, that both positive and negative regulators were represented in the GO enriched categories (Figure 3). Therefore, a more detailed analysis was required to determine if the pathways represented by the GO-enriched categories were promoted or inhibited during progression to CRPC. Moreover, many of the GO enrichments that were consistent with changes in the AR signaling pathway were generic, and could be applied to the other models of CRPC.

\section{Consistent differential gene expression associated with progression of prostate cancer}

Pair-wise comparisons were made between LongSAGE libraries representing the transcriptomes of different stages (AS, RAD, and CR) of prostate cancer progression from the same biological replicate (3 mice: $13 \mathrm{~N}, 15 \mathrm{~N}$, or $13 \mathrm{R}$ ). Among all three biological replicates, the number of consistent statistically significant differentially expressed tag types were determined using the Audic and Claverie test statistic [36] at $\mathrm{p} \leq 0.05, \mathrm{p} \leq 0.01$, and $\mathrm{p} \leq 0.001$ (Table 2). The tags represented in Table 2 were included only if the associated expression trend was common among all three biological replicates. The Audic and Claverie statistical method is well-suited for LongSAGE data, because the method takes into account the sizes of the libraries and tag counts. Tag types were counted multiple times if they were over, or under-represented in more than one comparison. The number of tag types differentially expressed decreased by $57 \%$ as the stringency of the p-value increased from $\mathrm{p} \leq 0.05$ to 0.001 .

Tag types consistently differentially expressed in pairwise comparisons were mapped to RefSeq (March 4th, 2008). Tags that mapped anti-sense to genes, or mapped ambiguously to more than one gene were not included in the functional analysis. GO, Kyoto Encyclopedia of Genes and Genomes (KEGG; v45.0) [37] pathway, and SwissProt (v13.0) [38] keyword annotation enrichment analyses were conducted using EASE (v1.21; March $11^{\text {th }}, 2008$ ) and FatiGO (v3; March $11^{\text {th }}, 2008$ ) [39] (Table 3). This functional analysis revealed that the expression of genes 


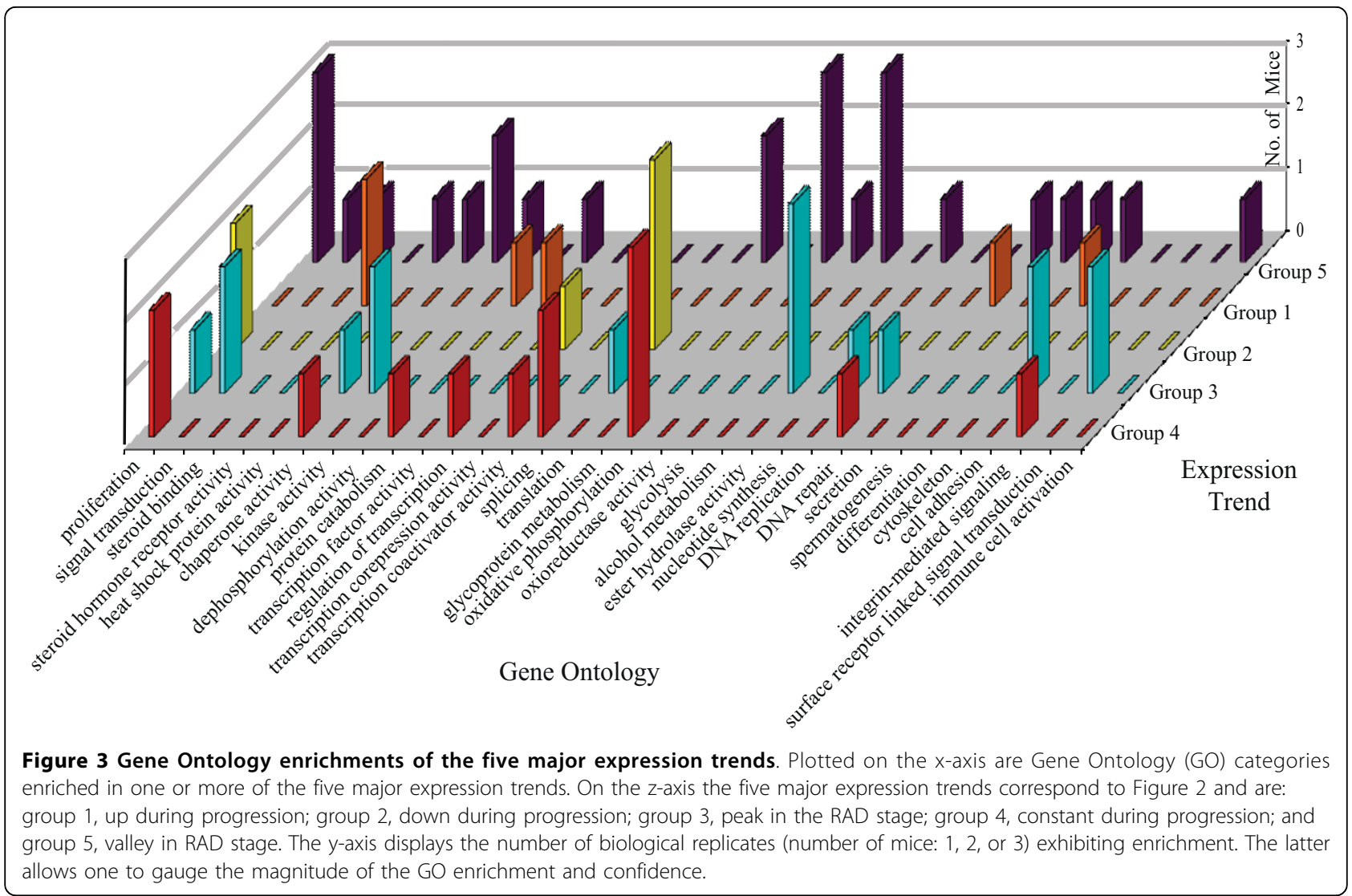

involved in signaling increased during progression, but the expression of genes involved in protein synthesis decreased during progression. Cell communication increased in the stage that was RAD but leveled off in the stage that was CR. Carbohydrate, lipid and amino acid

Table 2 Number of tag types consistently and significantly differentially expressed among all three biological replicates and between conditions*

\begin{tabular}{|c|c|c|c|c|}
\hline Comparison & Change & $p \leq 0.001$ & $p \leq 0.01$ & $p \leq 0.05$ \\
\hline \multirow[t]{3}{*}{ ASt vs. RAD $\neq$} & Up in RAD & 21 & 44 & 83 \\
\hline & Down in RAD & $\underline{68}$ & 105 & 149 \\
\hline & Total & 89 & $\overline{149}$ & $\overline{232}$ \\
\hline \multirow[t]{3}{*}{ RAD vs. CR§ } & Up in CR & 24 & 45 & 89 \\
\hline & Down in $C R$ & $\underline{46}$ & $\underline{59}$ & 104 \\
\hline & Total & 70 & $\overline{104}$ & $\overline{193}$ \\
\hline \multirow[t]{3}{*}{ AS vs. CR } & Up in CR & 111 & 167 & 294 \\
\hline & Down in $C R$ & 127 & 168 & 256 \\
\hline & Total & 238 & 335 & 550 \\
\hline
\end{tabular}

* Statistics according to the Audic and Claverie test statistic.

+ AS, Androgen-sensitive.

\# RAD, Responsive to androgen-deprivation.

$\S C R$, Castration-recurrent. synthesis was steady in the RAD stage but increased in the CR stage. Lastly, glycolysis decreased in the RAD stage, but was re-expressed in the CR stage (Table 3).

Tag types differentially expressed between the RAD and CR stages of prostate cancer were of particular interest (Table 4). This is because these tags potentially represent markers for CRPC and/or are involved in the mechanisms of progression to CRPC. These 193 tag types (Table 2) were mapped to databases RefSeq (July $9^{\text {th }}, 2007$ ), Mammalian Gene Collection (MGC; July $9^{\text {th }}, 2007$ ) [40], or Ensembl Transcript or genome (v45.36d) [41]. Only 135 of the 193 tag types were relevant (Table 4) with 48 tag types that mapped ambiguously to more than one location in the Homo Sapiens transcriptome/genome, and another 10 tag types that mapped to Mus musculus transcriptome/ genome. Mus musculus mappings may be an indication of minor contamination of the in vivo LNCaP Hollow Fiber model samples with host (mouse) RNA. These 135 tag types represented 114 candidate genes with 7 tag types that did not map to the genome, 5 tag types that mapped to unannotated genomic locations, and 9 genes that were associated with more than one tag type. Table 4 shows the LongSAGE tag sequences and tag counts per million tags in all nine libraries. Tags were sorted into groups based on expression trends. These trends are visually represented in 
Table 3 Top five enrichments of functional categories of tags consistently and significantly differentially expressed among all three biological replicates and between stages of prostate cancer*

\begin{tabular}{|c|c|c|c|c|c|}
\hline Top 5 GO + categories & $\begin{array}{l}\text { P-value } \\
\quad \neq\end{array}$ & Top 5 KEGG § annotations & $\begin{array}{c}\text { P-value } \\
\text { II }\end{array}$ & Top 5 SwissProt annotations & $\begin{array}{c}\text { P-value } \\
\text { II }\end{array}$ \\
\hline \multicolumn{6}{|c|}{ AS vs. RAD: Up in RAD } \\
\hline Cell communication & 2.E-02 & Stilbene, coumarine and lignin biosynthesis & 1.E-02 & Antioxidant & 7.E-04 \\
\hline Extracellular & 2.E-02 & Butanoate metabolism & 2.E-02 & Cell adhesion & 5.E-03 \\
\hline Extracellular matrix & 2.E-02 & 2,4-Dichlorobenzoate degradation & 2.E-02 & Signal & 6.E-03 \\
\hline Synaptic vesicle transport & 3.E-02 & Cell adhesion molecules (CAMs) & 2.E-02 & Fertilization & 7.E-03 \\
\hline Synapse & 4.E-02 & Alkaloid biosynthesis ॥ & 5.E-02 & Amyotrophic lateral sclerosis & 7.E-03 \\
\hline \multicolumn{6}{|c|}{ AS vs. RAD: Down in RAD } \\
\hline Glycolysis & 3.E-05 & Glycolysis/Gluconeogenesis & 3.E-05 & Glycolysis & 3.E-07 \\
\hline Glucose catabolism & 1.E-04 & Ribosome & 2.E-03 & Pyrrolidone carboxylic acid & 8.E-05 \\
\hline Hexose catabolism & 1.E-04 & Carbon fixation & 3.E-03 & Pyridoxal phosphate & 2.E-04 \\
\hline Hexose metabolism & 2.E-04 & Fructose and mannose metabolism & 2.E-02 & Gluconeogenesis & 3.E-04 \\
\hline Monosaccharide catabolism & 2.E-04 & Urea cycle and metabolism of amino groups & 3.E-02 & Coiled coil & 5.E-03 \\
\hline \multicolumn{6}{|c|}{ RAD vs. CR: Up in CR } \\
\hline Acid phosphatase activity & 4.E-02 & gamma-Hexachlorocyclohexane degradation & 5.E-03 & Lyase & 2.E-03 \\
\hline Lyase activity** & 7.E-02 & Glycolysis/Gluconeogenesis & 3.E-02 & Immune response & 5.E-03 \\
\hline Carbohydrate metabolism** & 9.E-02 & O-Glycan biosynthesis & 5.E-02 & Signal & 6.E-03 \\
\hline Extracellular** & 1.E-01 & Ether lipid metabolism** & 6.E-02 & Glycolysis & 7.E-03 \\
\hline Catabolism** & 1.E-01 & $\begin{array}{l}\text { Phenylalanine, tyrosine and tryptophan } \\
\text { biosynthesis** }\end{array}$ & 6.E-02 & $\begin{array}{l}\text { Progressive external } \\
\text { ophthalmoplegia }\end{array}$ & 1.E-02 \\
\hline \multicolumn{6}{|c|}{ RAD vs. CR: Down in CR } \\
\hline Cytosolic ribosome & 2.E-09 & Ribosome & 2.E-11 & Ribosomal protein & 6.E-10 \\
\hline Large ribosomal subunit & 1.E-07 & Urea cycle and metabolism of amino groups & 1.E-02 & Ribonucleoprotein & 3.E-08 \\
\hline Cytosol & 2.E-07 & Arginine and proline metabolism & 4.E-02 & Acetylation & 1.E-05 \\
\hline $\begin{array}{l}\text { Cytosolic large ribosomal } \\
\text { subunit }\end{array}$ & 2.E-07 & Type II diabetes mellitus** & 1.E-01 & Elongation factor & 1.E-03 \\
\hline Protein biosynthesis & 2.E-07 & Phenylalanine metabolism** & 1.E-01 & rRNA-binding & 2.E-03 \\
\hline \multicolumn{6}{|c|}{ AS vs. CR: Up in CR } \\
\hline Synapse & 4.E-03 & Butanoate metabolism & 2.E-03 & Glycoprotein & 2.E-03 \\
\hline Extracellular & 5.E-03 & Ascorbate and aldarate metabolism & 2.E-02 & Vitamin C & 7.E-03 \\
\hline Transition metal ion binding & 7.E-03 & Phenylalanine metabolism & 2.E-02 & Lipoprotein & 1.E-02 \\
\hline Metal ion binding & 2.E-02 & Linoleic acid metabolism & 2.E-02 & Signal & 1.E-02 \\
\hline Extracellular matrix & 2.E-02 & gamma-Hexachlorocyclohexane degradation & 2.E-02 & Heparin-binding & 1.E-02 \\
\hline \multicolumn{6}{|c|}{ AS vs. CR: Down in CR } \\
\hline Cytosolic ribosome & 4.E-12 & Ribosome & 2.E-09 & Acetylation & 2.E-07 \\
\hline Biosynthesis & 7.E-11 & Carbon fixation & 9.E-04 & Ribosomal protein & 1.E-06 \\
\hline Macromolecule biosynthesis & 2.E-10 & Glycolysis/Gluconeogenesis & 3.E-03 & Glycolysis & 7.E-05 \\
\hline Protein biosynthesis & 1.E-08 & Glycosphingolipid biosynthesis - lactoseries & 4.E-02 & Ribonucleoprotein & 8.E-05 \\
\hline $\begin{array}{l}\text { Eukaryotic } 43 \mathrm{~S} \text { preinitiation } \\
\text { complex }\end{array}$ & 2.E-08 & Glutamate metabolism** & 8.E-02 & Protein biosynthesis & 1.E-04 \\
\hline
\end{tabular}

* Statistics according to the Audic and Claverie test statistic $(p \leq 0.05)$.

† GO, Gene Ontology.

₹ P-value represents the raw EASE (Expression Analysis Systematic Explorer) score.

$\S$ KEGG, Kyoto Encyclopedia of Genes and Genomes.

II Unadjusted p-value was computed using FatiGO.

I $A S$, androgen-sensitive; RAD, responsive to androgen-deprivation; $C R$, castration-recurrent.

** Not statistically significant $(p>0.05)$. 
Table 4 Gene expression trends of LongSAGE tags that consistently and significantly altered expression in CR prostate cancer*

\begin{tabular}{|c|c|c|c|c|c|c|c|c|c|c|c|c|}
\hline \multirow[b]{3}{*}{ Tag Sequence } & \multicolumn{3}{|c|}{$13 N$} & \multicolumn{3}{|c|}{$15 N$} & \multicolumn{3}{|c|}{$13 R$} & \multirow{3}{*}{ Trend‡ } & \multirow[b]{3}{*}{ Gene** } & \multirow[b]{3}{*}{ Accession§§ } \\
\hline & AS§ & RADII & CRף & AS & RAD & CR & AS & RAD & CR & & & \\
\hline & S1885 & $\mathrm{S} 1886$ & S1887 & S1888 & S1889 & $\mathrm{S} 1890$ & S1891 & $\mathrm{S} 1892$ & S1893 & & & \\
\hline TCTAGAGAACACTGTGC & $12+$ & 79 & 382 & 7 & 67 & 136 & 7 & 52 & 200 & A & $A C P P \neq \neq$ & NM_001099 \\
\hline TAATTITTCTAAGGTGT & 101 & 311 & 648 & 119 & 397 & 895 & 120 & 546 & 918 & A & C1ORF80 & ENSG00000186063 \\
\hline TGAGAGAGGCCAGAACA & 8 & 39 & 150 & 4 & 39 & 144 & 7 & 33 & 95 & A & N/A & Genomic \\
\hline CTCATAAGGAAAGGTTA & 637 & 952 & 1680 & 653 & 1170 & 1540 & 688 & 1620 & 1930 & A & RNF208 & BC090061 \\
\hline GATTTCTATTTGTTIT & 89 & 169 & 446 & 116 & 208 & 339 & 86 & 311 & 555 & A & SERINC5 & ENSG00000164300 \\
\hline GTTGGGAAGACGTCACC & 426 & 571 & 742 & 273 & 417 & 741 & 262 & 363 & 495 & A & STEAP1 & NM_012449 \\
\hline GAGGATCACTTGAGGCC & 191 & 299 & 449 & 134 & 189 & 589 & 187 & 203 & 314 & B & $A M A C R \neq \neq$ & BC009471 \\
\hline TTGTTGATTGAAAATTT & 219 & 197 & 528 & 273 & 197 & 479 & 232 & 391 & 586 & B & AMD1㧊 & NM_001634 \\
\hline TTGCTITGTTTGTT & 53 & 16 & 169 & 34 & 51 & 129 & 7 & 28 & 72 & B & $A Q P 3$ & NM_004925 \\
\hline GTTCGACTGCCCACCAG & 45 & 28 & 101 & 52 & 47 & 122 & 34 & 42 & 106 & B & ASAH1†† & NM_177924 \\
\hline TAATAAACAGGTTITTA & 426 & 232 & 648 & 332 & 315 & 700 & 138 & 250 & 491 & B & ASAH1 $\neq \neq$ & NM_177924 \\
\hline TCACAGCTGTGAAGATC & 85 & 110 & 277 & 161 & 71 & 258 & 310 & 438 & 945 & B & BTG1 & NM_001731 \\
\hline AAAAGAGAAAGCACTIT & 24 & 75 & 199 & 19 & 35 & 85 & 15 & 90 & 552 & B & CAMK2N1 & NM_018584 \\
\hline CAAAACAGGCAGCTGGT & 4 & 71 & 169 & 15 & 83 & 162 & 37 & 75 & 268 & B & CAMK2N1+† & NM_018584 \\
\hline AGGAGGAAGAATGGACT & 33 & 59 & 187 & 49 & 67 & 247 & 26 & 42 & 223 & B & $\mathrm{CCNH}$ & NM_001239 \\
\hline TाTAAAAAATATAAAAT & 89 & 83 & 243 & 97 & 130 & 269 & 64 & 170 & 382 & B & COMT & NM_000754 \\
\hline GAATGAAATAAAAAATA & 134 & 252 & 626 & 209 & 240 & 357 & 116 & 160 & 272 & B & DHRS7 & NM_016029 \\
\hline AAAGTGCATCCTTTCCC & 118 & 146 & 318 & 153 & 220 & 394 & 288 & 231 & 646 & B & FGFRL1 & NM_001004356 \\
\hline AAACTGAATAAGGAGAA & 24 & 51 & 236 & 19 & 51 & 438 & 19 & 146 & 283 & B & GALNT3 & NM_004482 \\
\hline TTTAAGGAAACATTTGA & 4 & 4 & 75 & 4 & 4 & 81 & 0 & 0 & 57 & B & GALNT3+† & NM_004482 \\
\hline CCAACCGTGCTTGTACT & 191 & 327 & 521 & 202 & 279 & 534 & 172 & 363 & 510 & B & GLO1 & NM_006708 \\
\hline GAGGGCCGGTGACATCT & 300 & 378 & 1170 & 321 & 476 & 1230 & 254 & 447 & 1030 & B & H2AFJ & NM_177925 \\
\hline TATCATTATTITTACAA & 57 & 63 & 161 & 67 & 63 & 181 & 75 & 94 & 181 & B & HSD17B4 & NM_000414 \\
\hline AATGCACTTATGTTTGC & 16 & 8 & 64 & 22 & 16 & 77 & 19 & 28 & 98 & B & N/A & No map \\
\hline ACCTTCGCAGGGGAGAG & 0 & 0 & 19 & 0 & 4 & 41 & 0 & 5 & 34 & B & N/A & Genomic \\
\hline ATAACCTGAAAGGAAAG & 0 & 16 & 56 & 7 & 4 & 74 & 0 & 28 & 87 & B & N/A & No map \\
\hline GTGATGTGCACCTGTTG & 0 & 0 & 38 & 4 & 0 & 30 & 0 & 5 & 45 & B & N/A & No map \\
\hline GTTTGGAGGTACTAAAG & 20 & 43 & 94 & 34 & 87 & 169 & 34 & 90 & 234 & B & N/A & Genomic \\
\hline TTTCAAAAATTGGAAA & 0 & 35 & 180 & 7 & 4 & 59 & 0 & 19 & 61 & B & N/A & No map \\
\hline GAAAAATTTAAAGCTAA & 394 & 397 & 569 & 433 & 598 & 788 & 853 & 862 & 1060 & B & NGFRAP1 & NM_206917 \\
\hline CAAATTCAGGGAGCACA & 0 & 4 & 139 & 4 & 16 & 228 & 0 & 14 & 136 & B & OPRK1 & NM_000912 \\
\hline СTATTGTCTGAACTTGA & 0 & 8 & 109 & 0 & 12 & 70 & 0 & 9 & 227 & B & OR51E2 & BC020768 \\
\hline ATGCTAATTATGGCAAT & 4 & 12 & 75 & 4 & 8 & 74 & 0 & 5 & 57 & B & PCGEM1 & NR_002769 \\
\hline CAGAAAGCATCCCTCAC & 4 & 43 & 195 & 0 & 16 & 111 & 7 & 33 & 264 & B & PLA2G2A $\neq \neq$ & NM_000300 \\
\hline TAATITAATGCTTTGA & 16 & 75 & 154 & 37 & 59 & 162 & 4 & 57 & 132 & B & PTGFR & NM_000959 \\
\hline TTGTTTGTAAATAGAAT & 0 & 12 & 94 & 0 & 4 & 162 & 0 & 14 & 72 & B & QKI & NM_206853 \\
\hline TAAACACTGTAAAATCC & 0 & 4 & 75 & 0 & 4 & 66 & 0 & 0 & 42 & B & QKIt+ & NM_206853 \\
\hline AGCAGATCAGGACACTT & 20 & 35 & 112 & 15 & 16 & 140 & 15 & 42 & 98 & B & S100A10 & NM_002966 \\
\hline CTGCCATAACTTAGATT & 37 & 55 & 161 & 93 & 63 & 192 & 56 & 99 & 264 & B & SBDS & NM_016038 \\
\hline TGGCTGAGTTTATTITT & 20 & 24 & 79 & 41 & 8 & 96 & 4 & 42 & 147 & B & SFRS2B & NM_032102 \\
\hline GAAGATTAATGAGGGAA & 126 & 142 & 277 & 108 & 130 & 402 & 101 & 188 & 325 & B & SNX3 & NM_003795 \\
\hline ATGGTACTAAATGTTT & 16 & 47 & 124 & 37 & 28 & 88 & 11 & 19 & 76 & B & SPIRE1 & NM_020148 \\
\hline TATATATTAAGTAGCCG & 45 & 39 & 101 & 45 & 75 & 133 & 41 & 75 & 178 & B & STEAP2 $\neq \neq$ & NM_152999 \\
\hline CAACAATATATGCTTTA & 24 & 32 & 82 & 75 & 32 & 136 & 26 & 99 & 212 & B & STEAP2†† & NM_152999 \\
\hline TTTCATTGCCTGAATAA & 24 & 43 & 150 & 34 & 59 & 114 & 22 & 61 & 178 & B & TACC1 $\neq \neq$ & NM_006283 \\
\hline TTGGCCAGTCTGCTTTC & 8 & 16 & 67 & 4 & 4 & 77 & 0 & 5 & 38 & B & TMEM30A & ENSG00000112697 \\
\hline ATATCACTTCTTCTAGA & 12 & 4 & 26 & 7 & 4 & 26 & 0 & 52 & 140 & C & $A D A M 2 \neq \neq$ & NM_001464 \\
\hline ATGTGTGTTGTATTITA & 812 & 338 & 768 & 1010 & 315 & 1020 & 269 & 702 & 865 & C & BNIP3 & NM_004052 \\
\hline
\end{tabular}


Table 4: Gene expression trends of LongSAGE tags that consistently and significantly altered expression in CR prostate cancer* (Continued)

\begin{tabular}{|c|c|c|c|c|c|c|c|c|c|c|c|c|}
\hline CCACGTTCCACAGTTGC & 601 & 291 & 599 & 530 & 346 & 700 & 381 & 339 & 559 & C & ENO2 & NM_001975 \\
\hline CTGATCTGTGTTTCCTC & 16 & 0 & 26 & 0 & 4 & 41 & 19 & 0 & 34 & C & $H L A-B$ & BC013187 \\
\hline AGCCCTACAAACAACTA & 382 & 441 & 596 & 508 & 456 & 619 & 400 & 631 & 1010 & C & MT-ND3 & ENSG00000198840 \\
\hline ATATTTTCTTTGTGGAA & 20 & 12 & 90 & 7 & 0 & 48 & 4 & 0 & 23 & C & N/A & No map \\
\hline CAAGCATCCCCGTTCCA & 2400 & 2130 & 2440 & 2730 & 1720 & 2250 & 1020 & 2010 & 2340 & C & N/A & ENSG00000211459 \\
\hline GTTGTAAAATAAACTTT & 118 & 83 & 172 & 228 & 87 & 247 & 112 & 203 & 378 & C & N/A & Genoic \\
\hline TTGGATTTCCAAAGCAG & 12 & 0 & 19 & 0 & 0 & 33 & 0 & 0 & 26 & C & N/A & Genomic \\
\hline TCTTTTAGCCAATTCAG & 138 & 181 & 420 & 381 & 326 & 468 & 389 & 334 & 457 & C & NKX3-1†† & NM_006167 \\
\hline TGATTGCCCTTTCATAT & 73 & 39 & 86 & 86 & 39 & 107 & 108 & 99 & 181 & C & P4HA1 & NM_000917 \\
\hline GTAACAAGCTCTGGTAT & 28 & 16 & 56 & 49 & 24 & 66 & 11 & 19 & 72 & C & PJA2 & NM_014819 \\
\hline ACAGTGCTTGCATCCTA & 85 & 75 & 139 & 108 & 98 & 203 & 101 & 118 & 196 & C & $P P P 2 C B$ & NM_004156 \\
\hline AGGCGAGATCAATCCCT & 57 & 39 & 101 & 37 & 24 & 122 & 131 & 66 & 268 & C & PSMA7 & NM_002792 \\
\hline TATTTTGTATTTATTIT & 73 & 59 & 180 & 93 & 51 & 111 & 22 & 94 & 253 & C & SLC25A4 & NM_001151 \\
\hline TTATGGATCTCTCTGCG & 1050 & 1260 & 1820 & 1140 & 1300 & 2260 & 1990 & 1010 & 1530 & C & SPON2 & NM_012445 \\
\hline CAGTTCTCTGTGAAATC & 767 & 515 & 1060 & 855 & 503 & 914 & 467 & 608 & 1200 & C & TMEM66 & NM_016127 \\
\hline AAATAAATAATGGAGGA & 138 & 59 & 255 & 82 & 118 & 284 & 165 & 90 & 159 & C & TRPM8 & NM_024080 \\
\hline ATGTTAATTTTGCACA & 61 & 87 & 154 & 157 & 59 & 195 & 217 & 85 & 344 & C & WDR45L & NM_019613 \\
\hline GGGCCCCAAAGCACTGC & 861 & 543 & 1180 & 1020 & 657 & 1590 & 1240 & 739 & 937 & E & C19orf48 & NM_199249 \\
\hline TCCCCGTGGCTGTGGGG & 1670 & 1390 & 2290 & 1740 & 1410 & 1720 & 3370 & 970 & 1180 & E & DHCR24キ‡ & BC004375 \\
\hline GCATCTGTTACATTTA & 487 & 201 & 345 & 444 & 208 & 468 & 684 & 226 & 423 & E & ELOVL5 & NM_021814 \\
\hline GAAATTAGGGAAGCCTT & 317 & 153 & 311 & 310 & 181 & 542 & 359 & 193 & 298 & E & ENDOD1 & XM_290546 \\
\hline GGATGGGGATGAAGTAA & 2780 & 1160 & 4780 & 2950 & 1350 & 3620 & 2930 & 1230 & 1890 & E & $K L K 3 \neq \neq$ & NM_001648 \\
\hline TGAAAAGCTTAATAAAT & 313 & 142 & 322 & 474 & 181 & 332 & 273 & 179 & 314 & E & TPD52 & NM_001025252 \\
\hline GTTGTGGTTAATCTGGT & 1770 & 634 & 1270 & 1800 & 806 & 1190 & 2480 & 659 & 960 & $\mathrm{~F}$ & $B 2 M$ & NM_004048 \\
\hline GAAACAAGATGAAATTC & 4380 & 1170 & 2260 & 5300 & 1110 & 2720 & 3750 & 2220 & 2830 & $\mathrm{~F}$ & PGK1 & NM_000291 \\
\hline AGCACCTCCAGCTGTAC & 2150 & 1130 & 648 & 2060 & 1560 & 939 & 1560 & 1200 & 722 & G & EEF2 & NM_001961 \\
\hline GCACAAGAAGATTAAAA & 536 & 228 & 124 & 762 & 425 & 195 & 838 & 278 & 174 & G & GAS5 & NR_002578 \\
\hline CCGCTGCGTGAGGGCAG & 451 & 169 & 56 & 429 & 197 & 44 & 516 & 94 & 0 & G & HES6 & NM_018645 \\
\hline GCCCAGGTCACCCACCC & 585 & 55 & 4 & 519 & 79 & 7 & 456 & 66 & 0 & G & LOC644844 & XM_927939 \\
\hline ATGCAGCCATATGGAAG & 2650 & 386 & 82 & 2470 & 216 & 129 & 1210 & 259 & 98 & G & ODC1 & NM_002539 \\
\hline CGCTGGTTCCAGCAGAA & 1420 & 811 & 479 & 1250 & 959 & 553 & 800 & 589 & 374 & G & RPL11 & NM_000975 \\
\hline AAGACAGTGGCTGGCGG & 2650 & 1730 & 1220 & 2460 & 1860 & 1350 & 2120 & 1630 & 1270 & G & RPL37A $\neq \neq$ & NM_000998 \\
\hline TTCTTGTGGCGCTTCTC & 925 & 543 & 217 & 1030 & 708 & 273 & 1130 & 419 & 306 & G & RPS11十† & NM_001015 \\
\hline GGTGAGACACTCCAGTA & 463 & 252 & 165 & 485 & 346 & 192 & 363 & 245 & 159 & G & SLC25A6 & NM_001636 \\
\hline AGGTTTTGCCTCATTCC & 982 & 515 & 281 & 1200 & 491 & 243 & 688 & 782 & 166 & $\mathrm{H}$ & ABHD2 & NM_007011 \\
\hline TGAAGGAGCCGTCTCCA & 317 & 272 & 187 & 392 & 295 & 199 & 366 & 259 & 140 & $\mathrm{H}$ & ATP5G2 & NM_001002031 \\
\hline CTCAGCAGATCCAAGAG & 191 & 185 & 67 & 254 & 232 & 66 & 142 & 231 & 79 & $\mathrm{H}$ & C17orf45 & NM_152350 \\
\hline CTGTGACACAGCTTGCC & 308 & 397 & 172 & 209 & 307 & 125 & 295 & 226 & 110 & $\mathrm{H}$ & CCT2 & NM_006431 \\
\hline TCTGCACCTCCGCTTGC & 495 & 606 & 277 & 426 & 570 & 276 & 366 & 471 & 204 & $\mathrm{H}$ & EEF1A2 & NM_001958 \\
\hline GCCCAAGGACCCCCTGC & 114 & 114 & 38 & 138 & 98 & 41 & 101 & 42 & 4 & $\mathrm{H}$ & FLNA $\neq \ddagger$ & NM_001456 \\
\hline TTATGGGATCTCAACGA & 564 & 425 & 180 & 642 & 452 & 317 & 430 & 490 & 253 & $\mathrm{H}$ & GNB2L1 & NM_006098 \\
\hline TCTGCAAAGGAGAAGTC & 81 & 102 & 38 & 105 & 87 & 26 & 165 & 80 & 30 & $\mathrm{H}$ & HMGB2 & NM_002129 \\
\hline CTTGTGAACTGCACAAC & 268 & 228 & 124 & 231 & 177 & 103 & 273 & 160 & 57 & $\mathrm{H}$ & HN1 & NM_016185 \\
\hline TCTGAAGTTTGCCCCAG & 313 & 291 & 150 & 254 & 299 & 155 & 187 & 226 & 72 & $\mathrm{H}$ & $M A O A$ & NM_000240 \\
\hline TTAATTGATAGAATAAA & 483 & 350 & 199 & 422 & 287 & 103 & 273 & 235 & 83 & $\mathrm{H}$ & $M A O A$ & NM_000240 \\
\hline GGCAGCCAGAGCTCCAA & 1200 & 1260 & 420 & 1050 & 672 & 350 & 681 & 819 & 23 & $\mathrm{H}$ & MARCKSL1 & NM_023009 \\
\hline CCCTGCCTTGTCCCTCT & 353 & 240 & 112 & 310 & 263 & 107 & 176 & 193 & 102 & $\mathrm{H}$ & MDK & NM_001012334 \\
\hline CTGTGGATGTGTCCCCC & 649 & 476 & 169 & 459 & 389 & 214 & 430 & 297 & 117 & $\mathrm{H}$ & N/A & No map \\
\hline CTCCTCACCTGTATTIT & 1120 & 771 & 262 & 1220 & 979 & 313 & 666 & 730 & 261 & $\mathrm{H}$ & RPL13Aキキ & NM_012423 \\
\hline GCAGCCATCCGCAGGGC & 1980 & 1770 & 809 & 2300 & 1730 & 928 & 2150 & 1570 & 1020 & $\mathrm{H}$ & RPL28 & NM_000991 \\
\hline GGATTTGGCCTITTGA & 3470 & 2070 & 1370 & 4170 & 2910 & 1540 & 2800 & 2870 & 2500 & $\mathrm{H}$ & $R P L P 2 \neq \neq$ & NM_001004 \\
\hline
\end{tabular}


Table 4: Gene expression trends of LongSAGE tags that consistently and significantly altered expression in CR prostate cancer* (Continued)

\begin{tabular}{|c|c|c|c|c|c|c|c|c|c|c|c|c|}
\hline TCTGTACACCTGTCCCC & 2320 & 1670 & 850 & 1930 & 1880 & 825 & 2130 & 1490 & 1120 & $\mathrm{H}$ & RPS11 & NM_001015 \\
\hline GCTITTAAGGATACCGG & 1510 & 1050 & 626 & 1860 & 1120 & 593 & 1550 & 1550 & 960 & $\mathrm{H}$ & RPS20ł‡ & NM_001023 \\
\hline CCCCAGCCAGTCCCCAC & 921 & 519 & 281 & 788 & 664 & 357 & 1100 & 438 & 291 & H & RPS3 & NM_001005 \\
\hline CCCCCAATGCTGAGGCC & 89 & 138 & 26 & 90 & 94 & 30 & 90 & 80 & 30 & $\mathrm{H}$ & SF3A2 & NM_007165 \\
\hline GCCGCCATCTCCGAGAG & 195 & 102 & 30 & 168 & 118 & 55 & 172 & 108 & 30 & H & TKT & NM_001064 \\
\hline GGCCATCTCTTCCTCAG & 349 & 307 & 202 & 317 & 346 & 173 & 277 & 254 & 121 & $\mathrm{H}$ & YWHAQ & NM_006826 \\
\hline AGGCTGTGTTCCTCCGT & 16 & 39 & 11 & 34 & 67 & 22 & 26 & 38 & 8 & I & $A C Y 1$ & NM_000666 \\
\hline TGCCTCTGCGGGGCAGG & 446 & 649 & 427 & 399 & 664 & 424 & 501 & 462 & 317 & I & CD151 & NM_004357 \\
\hline GGCACAGTAAAGGTGGC & 175 & 216 & 142 & 332 & 350 & 173 & 456 & 316 & 204 & । & CUEDC2 & NM_024040 \\
\hline TCACACAGTGCCTGTCG & 49 & 71 & 7 & 30 & 47 & 15 & 34 & 66 & 4 & । & CXCR7 & NM_001047841 \\
\hline TGTGAGGGAAGCTGCTT & 53 & 87 & 15 & 67 & 102 & 52 & 52 & 90 & 42 & । & FKBP10 & BC016467 \\
\hline TGCTTTGCTTCATTCTG & 28 & 63 & 26 & 22 & 79 & 26 & 49 & 118 & 61 & । & GRB10 & NM_005311 \\
\hline GTACTGTATGCTTGCCA & 170 & 212 & 82 & 134 & 153 & 88 & 123 & 188 & 113 & । & KPNB1 キキ & NM_002265 \\
\hline GTGGCAGTGGCCAGTTG & 106 & 193 & 97 & 123 & 173 & 96 & 94 & 137 & 76 & । & N/A & ENSG00000138744 \\
\hline GGGGAGCCCCGGGCCCG & 61 & 63 & 26 & 30 & 51 & 18 & 34 & 57 & 0 & । & NAT14 & NM_020378 \\
\hline TGTTCAGGACCCTCCCT & 28 & 67 & 26 & 60 & 63 & 26 & 60 & 28 & 0 & । & NELF & NM_015537 \\
\hline TाTTCCTGGGGATCCTC & 41 & 130 & 15 & 37 & 87 & 33 & 56 & 104 & 45 & । & РCOTH & NM_001014442 \\
\hline GAAACCCGGTAGTCTAG & 41 & 75 & 4 & 37 & 75 & 26 & 52 & 151 & 30 & । & PLCB4 & NM_000933 \\
\hline GTCTGACCCCAGGCCCC & 126 & 205 & 82 & 119 & 193 & 103 & 157 & 179 & 38 & । & PPP2R1A & NM_014225 \\
\hline GGCCCGAGTTACTITTC & 231 & 150 & 75 & 161 & 232 & 136 & 142 & 160 & 45 & । & RPL35At† & NM_000996 \\
\hline GTTCGTGCCAAATTCCG & 881 & 696 & 390 & 1100 & 712 & 523 & 497 & 782 & 461 & । & RPL35A \#キ & NM_000996 \\
\hline TTACCATATCAAGCTGA & 877 & 535 & 311 & 1130 & 598 & 405 & 636 & 791 & 578 & । & RPL39キキ & NM_001000 \\
\hline GCTGCAGCACAAGCGGC & 268 & 244 & 127 & 45 & 216 & 125 & 157 & 71 & 11 & । & RPS18t+ & NM_022551 \\
\hline AGCTCTTGGAGGCACCA & 203 & 319 & 206 & 142 & 421 & 243 & 269 & 259 & 162 & । & SELENBP1 & NM_003944 \\
\hline TGCTGGTGTGTAAGGGG & 69 & 102 & 45 & 82 & 87 & 37 & 105 & 75 & 30 & । & SH3BP5L & NM_030645 \\
\hline GAGAGTAACAGGCCTGC & 191 & 150 & 71 & 112 & 181 & 111 & 108 & 165 & 64 & । & SYNC1 & NM_030786 \\
\hline CTGAAAACCACTCAAAC & 394 & 508 & 225 & 306 & 547 & 236 & 310 & 381 & 200 & । & TFPl & NM_006287 \\
\hline TAAAAAAGGTTTCATCC & 183 & 248 & 127 & 86 & 130 & 66 & 142 & 268 & 87 & । & TFPI & NM_006287 \\
\hline СТСССТССТСТССТАСС & 28 & 32 & 4 & 30 & 39 & 7 & 71 & 24 & 0 & । & TK1 & NM_003258 \\
\hline CATTTTCTAATTTGTG & 544 & 744 & 236 & 407 & 771 & 181 & 288 & 664 & 185 & J & N/A & No map \\
\hline TGATTTCACTTCCACTC & 3480 & 5260 & 3910 & 3700 & 6110 & 3590 & 3040 & 5960 & 2600 & K & MT-CO3 & ENSG00000198938 \\
\hline TाTCTGTCTGGGGAAGG & 130 & 236 & 82 & 123 & 201 & 111 & 101 & 188 & 113 & K & PIK3CD & NM_005026 \\
\hline GCCGCTACTTCAGGAGC & 256 & 370 & 199 & 224 & 330 & 169 & 142 & 316 & 38 & K & RAMP1 & NM_005855 \\
\hline ATGGTTACACTTITGGT & 93 & 161 & 94 & 75 & 208 & 118 & 60 & 226 & 95 & K & UTX & NM_021140 \\
\hline CACTACTCACCAGACGC & 2820 & 3900 & 3020 & 2740 & 4290 & 2440 & 2620 & 3120 & 1260 & K & VPS13B+† & ENSG00000132549 \\
\hline CTAAGACTTCACCAGTC & 7120 & 11000 & 9730 & 6390 & 10900 & 8330 & 3610 & 8870 & 7850 & L & N/A & ENSG00000210082 \\
\hline
\end{tabular}

* Statistics according to the Audic and Claverie test statistic $(p \leq 0.05)$.

+ Tag count per 1 million = (observed tag count/total tags in the library) $\times 1,000,000$.

₹ Trends are visually represented from A to $\mathrm{P}$ in Additional file 1, Figure S3. In addition to $\mathrm{p}$-value considerations, significantly different trends were also required to display uniform directions of change in each biological replicate.

$\S$ AS, Androgen-sensitive.

II RAD, Responsive to androgen-deprivation.

१ $\mathrm{CR}$, Castration-recurrent.

** Human Genome Nomenclature Committee (HGNC)-approved gene names were used when possible. Non-HGNC-approved gene names were not italicized. t† Tag maps antisense to gene.

㧊 Gene is known to display this expression trend in castration-recurrence.

$\S \S$ Accession numbers were displayed following the priority (where available): RefSeq > Mammalian Gene Collection > Ensembl Gene. If the tag mapped to more than one transcript variant of the same gene, the accession number of the lowest numerical transcript variant was displayed.

Additional file 1, Figure S3. Mapping information was provided where available.

We cross-referenced these 114 candidate genes with 28 papers that report global gene expression analyses on tissue samples from men with 'castration-recurrent', 'androgen independent,' 'hormone refractory,' 'androgenablation resistant,' 'relapsed,' or 'recurrent' prostate cancer, or animal models of castration-recurrence [42-69]. 
The candidate genes were identified with HUGO Gene Nomenclature Committee (HGNC) approved gene names, aliases, descriptions, and accession numbers. The gene expression trends of 18 genes of 114 genes were previously associated with CRPC. These genes were: ACPP, ADAM2, AMACR, AMD1, ASAH1, DHCR24, FLNA, KLK3, KPNB1, PLA2G2A, RPL13A, RPL35A, RPL37A, RPL39, RPLP2, RPS20, STEAP2, and TACC (Table 4). To our knowledge, the gene expression trends of the remaining 96 genes have never before been associated with CRPC (Tables 4 \&5).

A literature search helped to gauge the potential of these 96 genes to be novel biomarkers or therapeutic targets of CRPC. The results of this literature search are presented in Table 5 . We found 31 genes that encode for protein products that are known, or predicted, to be plasma membrane bound or secreted extracellularly (Bioinformatic Harvester). These genes were: $A B H D 2, A Q P 3, B 2 M$, C19orf48, CD151, CXCR7, DHRS7, ELOVL5, ENDOD1, ENO2, FGFRL1, GNB2L1, GRB10, HLA-B, MARCKSL1, MDK, NAT14, NELF, OPRK1, OR51E2, PLCB4, PTGFR, RAMP1, S100A10, SPON2, STEAP1, TFPI, TMEM30A, TMEM66, TRPM8, and $V P S 13 B$. Secretion of a protein could facilitate detection of the putative biomarkers in blood, urine, or biopsy sample. Twenty-one of the candidate genes are known to alter their levels of expression in response to androgen. These genes were: $A B H D 2, B 2 M, B T G 1$, C19orf48, CAMK2N1, CXCR7, EEF1A2, ELOVL5, ENDOD1, HSD17B4, MAOA, MDK, NKX3-1, ODC1, P4HA1, PCGEM1, PGK1, SELENBP1, TMEM66, TPD52, and TRPM8 [9,22,70-81]. Genes regulated by androgen may be helpful in determining the activation status of AR in CRPC. Enriched expression of a protein in prostate tissue could be indicative of whether a tumor is of prostatic origin. Eight of these 96 genes are known to be over-represented in prostate tissue [75,82-85]. These genes were: ELOVL5, NKX3-1, PCGEM1, PCOTH, RAMP1, SPON2, STEAP1, and TPD52. Twenty-six genes (ABHD2, BNIP3, EEF1A2, ELOVL5, GALNT3, GLO1, HSD17B4, MARCKSL1, MDK, NGFRAP1, ODC1, OR51E2, PCGEM1, PCOTH, PGK1, PP2CB, PSMA7, RAMP1, RPS18, SELENBP1, SLC25A4, SLC25A6, SPON2, STEAP1, TPD52, and TRPM8) have known associations to prostate cancer [57,82,86-102]. Six genes (C1orf80, CAMK2N1, GLO1, $M A O A, P G K 1$, and $S N X 3$ ) have been linked to high Gleason grade $[58,103,104]$, and twelve genes (B2 $M$, CAMK2N1, CD151, COMT, GALNT3, GLO1, ODC1, PCGEM1, PCOTH, SBDS, TMEM30A, and TPD52) have been implicated in the 'progression' of prostate cancer [58,82], and 15 more genes (CD151, CXCR7, DHRS7, GNB2L1, HES6, HN1, NKX3-1, PGK1,
PIK3CD, RPL11, RPS11, SF3A2, TK1, TPD52, and $V P S 13 B)$ in the metastasis of prostate cancer $[105,106]$.

\section{Novel CR-associated genes identify both clinical samples of CRPC and clinical metastasis of prostate cancer}

The expression of novel CR-associated genes were validated in publically available, independent sample sets representing different stages of prostate cancer progression (Gene Expression Omnibus accession numbers: GDS1390 and GDS1439). Dataset GDS1390 includes expression data of ten AS prostate tissues, and ten CRPC tissues from Affymetrix U133A arrays [47]. Dataset GDS1439 includes expression data of six benign prostate tissues, seven localized prostate cancer tissues, and seven metastatic prostate cancer tissues from Affymetrix U133 2.0 arrays [97].

Unsupervised principal component analysis based on the largest three principal components revealed separate clustering of tumor samples representing AS and CR stages of cancer progression, with the exception of two CR samples and one AS sample (Figure 4a).

Metastatic prostate cancer is expected to have a more progressive phenotype and is associated with hormonal progression. Therefore, the gene expression signature obtained from the study of hormonal progression may be common to that observed in clinical metastases. Unsupervised principal component analysis based on the largest three principal components revealed separate clustering of not only benign and malignant, but also localized and metastatic tissue samples (Figure $4 b$ ).

\section{Discussion}

Genes that change levels of expression during hormonal progression may be indicative of the mechanisms involved in CRPC. Here we provide the most comprehensive gene expression analysis to date of prostate cancer with approximately 3 million long tags sequenced using in vivo samples of biological replicates at various stages of hormonal progression to improve over the previous libraries that are approximately 70,000 short tags or less. Previous large-scale gene expression analyses have been performed with tissue samples from men with advanced prostate cancer [42-58], and animal or xenograft models of CRPC [59-69]. Most of these previous studies compared differential expression between CRPC samples with the primary samples obtained before androgen ablation. This experimental design cannot distinguish changes in gene expression that are a direct response to androgen ablation, or from changes in proliferation/survival that have been obtained as the prostate cancer cells progress to more a more advanced phenotype. Here we are the first to apply an in vivo model of hormonal progression to compare gene 
Table 5 Characteristics of genes with novel association to castration-recurrence in vivo

\begin{tabular}{|c|c|c|c|c|c|c|c|c|c|c|c|c|c|c|c|c|c|}
\hline \multirow[b]{2}{*}{ Gene* } & \multirow[b]{2}{*}{$\begin{array}{l}\text { S or } \\
\text { PMt }\end{array}$} & \multirow[b]{2}{*}{$\begin{array}{c}\text { Reg. } \\
\text { by A }\end{array}$} & \multirow[b]{2}{*}{$\begin{array}{l}\text { Spec. } \\
\text { to P§ }\end{array}$} & \multicolumn{5}{|c|}{ Associated with } & \multirow[b]{2}{*}{ Gene } & \multirow[b]{2}{*}{$\begin{array}{l}\text { S or } \\
\text { PM }\end{array}$} & \multirow[b]{2}{*}{$\begin{array}{l}\text { Reg. } \\
\text { by A }\end{array}$} & \multirow[b]{2}{*}{$\begin{array}{l}\text { Spec. } \\
\text { to } P\end{array}$} & \multicolumn{5}{|c|}{ Associated with } \\
\hline & & & & CaPII & GGף & $\underset{* *}{\text { Prog. }}$ & Metst† & $\mathrm{CR} \neq \neq$ & & & & & $\mathrm{CaP}$ & GG & Prog. & Mets & $\mathrm{CR}$ \\
\hline ABHD2 & PM & $\overline{Y \uparrow}$ & - & $Y \uparrow$ & - & - & - & - & NKX3-1 & - & $\mathrm{Y \uparrow}$ & Y & - & - & - & Y & - \\
\hline$A C Y 1$ & - & - & - & - & - & - & - & - & ODC1 & - & $Y \uparrow$ & - & $Y \uparrow$ & - & $Y \downarrow$ & - & $\mathrm{Y} \uparrow$ \\
\hline$A Q P 3$ & PM & - & - & - & - & - & - & - & OPRK1 & PM & - & - & - & - & - & - & - \\
\hline ATP5G2 & - & - & - & - & - & - & - & - & OR51E2 & PM & - & - & $Y \uparrow$ & - & - & - & - \\
\hline$B 2 M$ & S\&PM & $Y \uparrow$ & - & - & - & $Y \uparrow$ & - & $Y \downarrow$ & P4HAT & - & Y & - & - & - & - & - & - \\
\hline BNIP3 & - & - & - & $Y \downarrow$ & - & - & - & - & PCGEM1 & - & $\mathrm{Y} \uparrow$ & Y & $Y \uparrow$ & - & $\mathrm{Y} \uparrow$ & - & - \\
\hline BTG1 & - & $Y \downarrow$ & - & - & - & - & - & - & PCOTH & - & - & Y & $Y \uparrow$ & - & $Y \uparrow$ & - & - \\
\hline C17orf45 & - & - & - & - & - & - & - & - & PGK1 & - & $Y \uparrow$ & - & $Y \downarrow$ & $Y \uparrow$ & - & $\begin{array}{c}Y \\
\uparrow \downarrow \S \S\end{array}$ & - \\
\hline C19orf48 & $S$ & $Y \uparrow$ & - & - & - & - & - & - & PIK3CD & - & - & - & - & - & - & $Y \uparrow$ & $Y \uparrow$ \\
\hline C1orf80 & - & - & - & - & $Y \uparrow$ & - & - & - & PJA2 & - & - & - & - & - & - & - & - \\
\hline CAMK2N1 & - & $Y \downarrow$ & - & - & $Y \uparrow$ & $Y \uparrow$ & - & - & PLCB4 & PM & - & - & - & - & - & - & - \\
\hline $\mathrm{CCNH}$ & - & - & - & - & - & - & - & - & PPP2CB & - & - & - & $Y \downarrow$ & - & - & - & - \\
\hline $\mathrm{CCT} 2$ & - & - & - & - & - & - & - & - & PPP2R1A & - & - & - & - & - & - & - & - \\
\hline CD151 & PM & - & - & - & - & $Y \uparrow$ & $Y \uparrow$ & - & PSMA7 & - & - & - & $Y \downarrow$ & - & - & - & - \\
\hline СOMT & - & - & - & - & - & $Y \downarrow$ & - & - & PTGFR & PM & - & - & - & - & - & - & - \\
\hline CUEDC2 & - & - & - & - & - & - & - & - & QKI & - & - & - & - & - & - & - & - \\
\hline CXCR7 & PM & $Y \downarrow$ & - & - & - & - & $Y \uparrow$ & $Y \uparrow$ & RAMP1 & PM & - & Y & $Y \uparrow$ & - & - & - & - \\
\hline DHRS7 & PM & - & - & - & - & - & $Y \downarrow$ & - & RNF208 & - & - & - & - & - & - & - & - \\
\hline EEF1A2 & - & $Y \uparrow$ & - & $Y \uparrow$ & - & - & - & - & RPL11 & - & - & - & - & - & - & $Y \downarrow$ & - \\
\hline EEF2 & - & - & - & - & - & - & - & - & RPL28 & - & - & - & - & - & - & - & - \\
\hline ELOVL5 & PM & Y & Y & $Y \downarrow$ & - & - & - & - & RPS11 & - & - & - & - & - & - & $Y \downarrow$ & - \\
\hline ENDOD1 & $S$ & $Y \uparrow$ & - & - & - & - & - & - & RPS18 & - & - & - & $Y \uparrow$ & - & - & - & - \\
\hline ENO2 & PM & - & - & - & - & - & - & - & RPS3 & - & - & - & - & - & - & - & - \\
\hline ENSG00000210082 & - & - & - & - & - & - & - & - & S100A10 & PM & - & - & - & - & - & - & - \\
\hline ENSG00000211459 & - & - & - & - & - & - & - & - & SBDS & - & - & - & - & - & $Y \uparrow$ & - & - \\
\hline FGFRL1 & PM & - & - & - & - & - & - & - & SELENBP1 & - & $Y \downarrow$ & - & $Y \downarrow$ & - & - & - & - \\
\hline FKBP10 & - & - & - & - & - & - & - & - & SERINC5 & - & - & - & - & - & - & - & - \\
\hline GALNT3 & - & - & - & $\mathrm{Y} \uparrow$ & - & $Y \downarrow$ & - & - & SF3A2 & - & - & - & - & - & - & $\mathrm{Y} \uparrow$ & - \\
\hline GAS5 & - & - & - & - & - & - & - & - & SFRS2B & - & - & - & - & - & - & - & - \\
\hline GLO1 & - & - & - & $Y \uparrow$ & $Y \uparrow$ & $Y \uparrow$ & - & - & SH3BP5L & - & - & - & - & - & - & - & - \\
\hline GNB2L1 & PM & - & - & - & - & - & $Y \uparrow$ & - & SLC25A4 & - & - & - & $Y \uparrow$ & - & - & - & - \\
\hline GRB10 & PM & - & - & - & - & - & - & - & SLC25A6 & - & - & - & $\mathrm{Y} \uparrow$ & - & - & - & - \\
\hline H2AFJ & - & - & - & - & - & - & - & - & SNX3 & - & - & - & - & $Y \uparrow$ & - & - & - \\
\hline HES6 & - & - & - & - & - & - & $Y \uparrow$ & $Y \uparrow$ & SPIRE1 & - & - & - & - & - & - & - & - \\
\hline$H L A-B$ & PM & - & - & - & - & - & - & - & SPON2 & $S$ & - & Y & $Y \uparrow$ & - & - & - & - \\
\hline HMGB2 & - & - & - & - & - & - & - & $\mathrm{Y} \uparrow$ & STEAP1 & PM & - & Y & $Y \uparrow$ & - & - & - & - \\
\hline HN1 & - & - & - & - & - & - & $Y \uparrow$ & - & SYNC1 & - & - & - & - & - & - & - & - \\
\hline HSD17B4 & - & $Y \uparrow$ & - & $Y \uparrow$ & - & - & - & - & TFPI & S & - & - & - & - & - & - & - \\
\hline LOC644844 & - & - & - & - & - & - & - & - & TK1 & - & - & - & - & - & - & $Y \uparrow$ & - \\
\hline$M A O A$ & - & Y & - & - & $Y \uparrow$ & - & - & - & TKT & - & - & - & - & - & - & - & - \\
\hline MARCKSL1 & PM & - & - & $Y \uparrow$ & - & - & - & - & TMEM3OA & S\&PM & - & - & - & - & $Y \uparrow$ & - & - \\
\hline MDK & S\&PM & $Y \downarrow$ & - & $Y \uparrow$ & - & - & - & $Y \uparrow$ & TMEM66 & S\&PM & $Y \uparrow$ & - & - & - & - & - & - \\
\hline MT-CO3 & - & - & - & - & - & - & - & - & TPD52 & - & $Y \uparrow$ & Y & $Y \uparrow$ & - & $Y \uparrow$ & $Y \downarrow$ & - \\
\hline MT-ND3 & - & - & - & - & - & - & - & - & TRPM8 & PM & $\mathrm{Y} \uparrow$ & - & $Y \uparrow$ & - & - & - & $Y \downarrow$ \\
\hline NAAA & - & - & - & - & - & - & - & $Y \uparrow$ & UTX & - & - & - & - & - & - & - & - \\
\hline NAT14 & PM & - & - & - & - & - & - & - & VPS13B & PM & - & - & - & - & - & $Y \uparrow$ & - \\
\hline
\end{tabular}


Table 5: Characteristics of genes with novel association to castration-recurrence in vivo (Continued)

\begin{tabular}{|c|c|c|c|c|c|c|c|c|}
\hline NELF & PM & - & - & - & - & - & - & WDR45 \\
\hline NGFRAP1 & - & - & - & $Y \downarrow$ & - & - & - & YWHAQ \\
\hline
\end{tabular}

* Human Genome Nomenclature Committee (HGNC)-approved gene names were used when possible. Non-HGNC-approved gene names were not italicized.

† $\mathrm{S}$ or $\mathrm{PM}$, gene product is thought to be secreted (S) or localize to the plasma membrane (PM).

₹ Reg. by $A$, gene expression changes in response to androgen in prostate cells.

$\S$ Spec. to $\mathrm{P}$, gene expression is specific to- or enriched in- prostate tissue compared to other tissues.

II CaP, gene is differentially expressed in prostate cancer compared to normal, benign prostatic hyperplasia, or prostatic intraepithelial neoplasia.

१ GG, gene is differentially expressed in higher Gleason grade tissue versus lower Gleason grade tissue.

** Prog., gene expression correlates with late-stage prostate cancer or is a risk factor that predicts progression.

†† Mets, gene expression is associated with prostate cancer metastasis in human samples or in vivo models.

¥ CR, gene is associated with castration-recurrent prostate cancer in human tissue or in vivo models, but exhibits an opposite trend of this report

$\S \S \mathrm{Y}$, yes; $\uparrow$, high gene expression; $\downarrow$, low gene expression.

expression between serial samples of prostate cancer before (AS), and after androgen ablation therapy (RAD) as well as when the cells become CR. This model is the LNCaP Hollow Fiber model [21] which has genomic similarity with clinical prostate cancer [23] and mimics the hormonal progression observed clinically in response to host castration as measured by levels of expression of PSA and cell proliferation. Immediately prior to castration, when the cells are AS, PSA levels are elevated and the LNCaP cells proliferate. A few days following castration, when the cells are RAD, PSA levels drop and the LNCaP cells cease to proliferate, but do not apoptose in this model. Approximately 10 weeks following castration, when the cells are CR, PSA levels rise and the LNCaP cells proliferate in the absence of androgen. This model overcomes some limitations in other studies using xenografts that include host contamination of prostate cancer cells. The hollow fibers prevent infiltration of host cells into the fiber thereby allowing retrieval of pure populations of prostate cells from within the fiber. The other important benefit of the fiber model is the ability to examine progression of cells to CRPC at various stages within the same host mouse over time, because the retrieval of a subset of fibers entails only minor surgery. The power to evaluate progression using serial samples from the same mouse minimizes biological variation to enhance the gene expression analyses. However, limitations of this model include the lack of cell-cell contact with stroma cells, and lack of heterogeneity in tumors. Typically, these features would allow paracrine interactions as expected in clinical situations. Consistent with the reported clinical relevance of this model [23], here principal component analysis based on the expression of these novel genes identified by LongSAGE, clustered the clinical samples of CRPC separately from the androgen-dependent
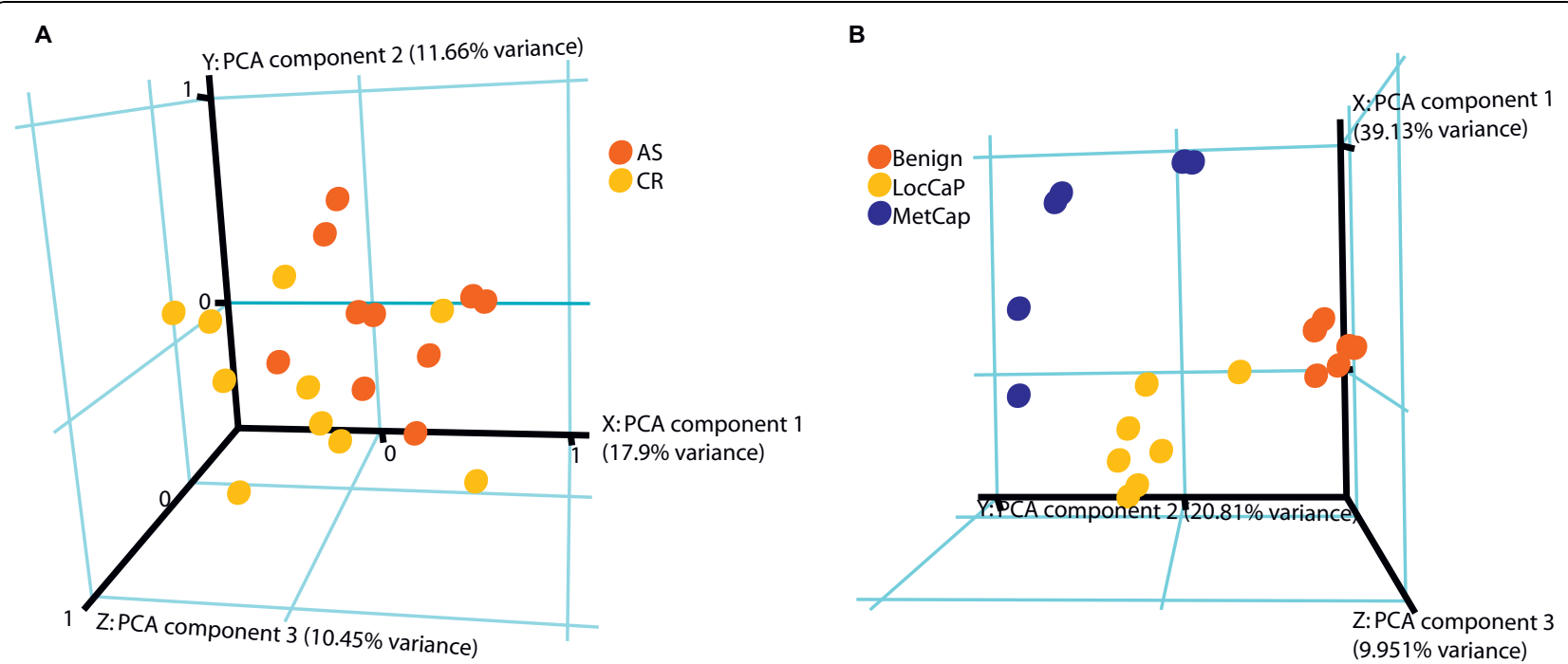

Figure 4 Principle component analyses of clinical samples. A, Principle component analysis based on the expression of novel CR-associated genes in the downloaded dataset GDS1390 clustered the AS and CR clinical samples into two groups. B, Principle component analysis based on the expression of novel CR-associated genes in the downloaded dataset GDS1439 clustered the clinical samples (benign prostate tissue, benign; localized prostate cancer, Loc CaP; and metastatic prostate cancer, Met CaP) into three groups. 
samples. Principal component analysis based on the expression of these genes also revealed separate clustering of the different stages of tumor samples and also showed separate clustering of the benign samples from the prostate cancer samples. Therefore, some common changes in gene expression profile may lead to the survival and proliferation of prostate cancer and contribute to both distant metastasis and hormonal progression. We used this LNCaP atlas to identify changes in gene expression that may provide clues of underlying mechanisms resulting in CRPC. Suggested models of CRPC involve: the AR; steroid synthesis and metabolism; neuroendocrine prostate cancer cells; and/or an imbalance of cell growth and cell death.

\section{Androgen receptor (AR) \\ Transcriptional activity of $A R$}

The AR is suspected to continue to play an important role in the hormonal progression of prostate cancer. The AR is a ligand-activated transcription factor with its activity altered by changes in its level of expression or by interactions with other proteins. Here, we identified changes in expression of some known or suspected modifier of transcriptional activity of the ARin CRPC versus RAD such as Cyclin $\mathrm{H}(C \mathrm{CNH})$ [107], proteasome macropain subunit alpha type 7 (PSMA7) [108], CUE-domain-containing-2 (CUEDC2) [109], filamin A (FLNA) [110], and high mobility group box 2 (HMGB2) [111]. CCNH and PSMA7 displayed increased levels of expression, while CUEDC2, FLNA, and HMGB2 displayed decreased levels of expression in CR. The expression trends of CCNH, CUEDC2, FLNA, and PSMA7 in CRPC may result in increased AR signaling through mechanisms involving protein-protein interactions or altering levels of expression of AR. $\mathrm{CCNH}$ protein is a component of the cyclin-dependent activating kinase (CAK). CAK interacts with the AR and increases its transcriptional activity [107]. Over-expression of the proteosome subunit PSMA7 promotes AR transactivation of a PSA-luciferase reporter [108]. A fragment of the protein product of FLNA negatively regulates transcription by AR through a physical interaction with the hinge region [110]. CUEDC2 protein promotes the degradation of progesterone and estrogen receptors [109]. These steroid receptors are highly related to the $\mathrm{AR}$, indicating a possible role for CUEDC2 in AR degradation. Thus decreased expression of FLNA or CUEDC2 could result in increased activity of the AR. Decreased expression of HMGB2 in CRPC is predicted to decrease expression of at least a subset of androgen-regulated genes that contain palindromic AREs [111]. Here, genes known to be regulated by androgen were enriched in expression trend categories with a peak or valley at the RAD stage of prostate cancer progression. Specifically, 8 of the 13 tags (62\%) exhibiting these expression trends 'E', 'F', 'J', 'K', or 'L' represented known androgenregulated genes, in contrast to only 22 of the remaining 122 tags (18\%; Tables 4 \&5). Overall, this data supports increased AR activity in CRPC, which is consistent with re-expression of androgen-regulated genes as previously reported [68] and similarity of expression of androgen regulated genes between CRPC and prostate cancer before androgen ablation [23].

\section{Steroid synthesis and metabolism}

In addition to changes in expression of AR or interacting proteins altering the transcriptional activity of the AR, recent suggestion of sufficient levels of residual androgen in CRPC provides support for an active ligand-bound receptor [112]. The AR may become reactivated in CRPC due to the presence of androgen that may be synthesized by the prostate de novo [4] or through the conversion of adrenal androgens. Here, the expression of 5 genes known to function in steroid synthesis or metabolism were significantly differentially expressed in CRPC versus RAD. They are 24-dehydrocholesterol reductase (DHCR24) [113], dehydrogenase/ reductase SDR-family member 7 (DHRS7) [114], elongation of long chain fatty acids family member 5 (ELOVL5) [115,116], hydroxysteroid (17-beta) dehydrogenase 4 (HSD17B4) [117], and opioid receptor kappa 1 (OPRK1) [118]. Increased levels of expression of these genes may be indicative of the influence of adrenal androgens, or the local synthesis of androgen, to reactivate the AR to promote the progression of prostate cancer in the absence of testicular androgens.

\section{Neuroendocrine}

Androgen-deprivation induces neuroendocrine differentiation of prostate cancer. Here, the expression of 8 genes that are associated with neuroendocrine cells were significantly differentially expressed in CRPC versus RAD. They either responded to androgen ablation such as hairy and enhancer of split 6 (HES6) [119], karyopherin/importin beta 1 (KPNB1) [120], monoamine oxidase A $(M A O A)$ [121], and receptor (calcitonin) activity modifying protein 1 (RAMP1) [122]], or were increased expressed in CRPC such as ENO2 [122], OPRK1 [118], S100 calcium binding protein A10 (S100A10) [123], and transient receptor potential cation channel subfamily $M$ member 8 (TRPM8) [124].

\section{Proliferation and Cell survival}

The gene expression trends of GAS5 [125], GNB2L1 [126], MT-ND3, NKX3-1 [127], PCGEM1 [128], PTGFR [129], STEAP1 [130], and TMEM30A [131] were in agreement with the presence of proliferating cells in CRPC. Of particular interest is that we observed a transcript anti-sense to $N K X 3-1$, a tumor suppressor, 
highly expressed in the stages of cancer progression that were AS and CR, but not RAD. Anti-sense transcription may hinder gene expression from the opposing strand, and therefore, represents a novel mechanism by which NKX3-1 expression may be silenced. There were also some inconsistencies including the expression trends of BTG1 [132], FGFRL1 [133], and PCOTH [134] and that may be associated with non-cycling cells. Overall, there was more support at the transcriptome level for proliferation than not, which was consistent with increased proliferation observed in the LNCaP Hollow Fiber model [21].

Gene expression trends of GLO1 [135], S100A10 [136], TRPM8 [137], and PI3KCD [138] suggest cell survival pathways are active following androgen-deprivation and/ or in CRPC, while gene expression trends of CAMK2N1 [139], CCT2 [140], MDK [141,142], TMEM66 [143], and $Y W H A Q$ [136] may oppose such suggestion. Taken together, these data neither agree nor disagree with the activation of survival pathways in CRPC. In contrast to earlier reports in which $M D K$ gene and protein expression was determined to be higher in late stage cancer [63,142], we observed a drop in the levels of $M D K$ mRNA in CRPC versus RAD. $M D K$ expression is negatively regulated by androgen [65]. Therefore, the decreased levels of $M D K$ mRNA in CRPC may suggest that the AR is reactivated in CRPC.

\section{Other}

The significance of the gene expression trends of AMD1, BNIP3, GRB10, MARCKSL1, NGRAP1, ODC1, PPP2CB, PPP2R1A, SLC25A4, SLC25A6, and WDR45L that function in cell growth or cell death/survival were not straightforward. For example, BNIP3 and WDR45L, both relatively highly expressed in CRPC versus RAD, may be associated with autophagy. BNIP3 promotes autophagy in response to hypoxia [144], and the WDR45L-related protein, WIPI-49, co-localizes with the autophagic marker LC3 following amino acid depletion in autophagosomes [145]. It is not known if BNIP3 or putative WDR45L-associated autophagy results in cell survival or death. Levels of expression of NGFRAP1 were increased in CRPC versus RAD. The protein product of NGFRAP1 interacts with p75 (NTR). Together they process caspase 2 and caspase 3 to active forms, and promote apoptosis in 293T cells [146]. NGFRAP1 requires p75 (NTR) to induce apoptosis. However, LNCaP cells do not express p75 (NTR), and so it is not clear if apoptosis would occur in this cell line [147].

Overall, genes involved in cell growth and cell death pathways were altered in CRPC. Increased tumor burden may develop from a small tip in the balance when cell growth outweighs cell death. Unfortunately, the contributing weight of each gene is not known, making predictions difficult based on gene expression alone of whether proliferation and survival were represented more than cell death in this model of CRPC. It should be noted that LNCaP cells are androgen-sensitive and do not undergo apoptosis in the absence of androgens. The proliferation of these cells tends to decrease in androgen-deprived conditions, but eventually with progression begins to grow again mimicking clinical CRPC.

\section{Conclusion}

Here, we describe the LNCaP atlas, a compilation of LongSAGE libraries that catalogue the transcriptome of human prostate cancer cells as they progress to CRPC in vivo. Using the LNCaP atlas, we identified differential expression of 96 genes that were associated with castration-recurrence in vivo. These changes in gene expression were consistent with the suggested model for a role of the AR, steroid synthesis and metabolism, neuroendocrine cells, and increased proliferation in CRPC.

\section{Additional material}

\begin{abstract}
Additional file 1: Supplementary Figures. Figure S1: qRT-PCR analysis of $K L K 3$ gene expression during hormonal progression of prostate cancer to castration-recurrence. RNA samples were retrieved from the in vivo LNCaP Hollow Fiber model at different stages of cancer progression that were: AS, androgen-sensitive, day zero (just prior to surgical castration and 7 days post-fiber implantation); RAD, responsive to androgendeprivation, 10 days post-surgical castration; and CR, castration-recurrent, 72 days post-surgical castration. MNE, mean normalized expression, calculated by normalization to glyceraldehyde-3-phosphate (GAPDH). Error bars represent \pm standard deviation of technical triplicates. Each mouse represents one biological replicate. Figure S2: Ten K-means clusters are optimal to describe the expression trends present during progression to castration-recurrence. K-means clustering was conducted over a range of $\mathrm{K}$ (number of clusters) from $\mathrm{K}=2$ to $\mathrm{K}=20$ and the within-cluster dispersion was computed for each clustering run and plotted against $\mathrm{K}$. The within-cluster dispersion declined with the addition of clusters and this decline was most pronounced at $K=10$. The graph of within cluster dispersion versus $\mathrm{K}$ shown here is for mouse $13 \mathrm{~N}$, but the results were similar for mice $15 \mathrm{~N}$ and $13 \mathrm{R}$. Figure S3: Trend legend for Table 4. Gene expression trends of LongSAGE tags that consistently and significantly altered expression in CR prostate cancer are represented graphically with trends labeled A-P. * Statistics according to the Audic and Claverie test statistic ( $p \leq 0.05)$.
\end{abstract}

\section{Abbreviations}

ACPP: prostate acid phosphatise; ACTH: adrenocorticotropic hormone; AR: androgen receptor; AREs: androgen response elements; AS: androgensensitive; $B A X$ : BCL2-associated X protein; $B C L-2$ : B-cell CLL/lymphoma 2; BCL2L1: BCL2-like 1; CAK: cyclin-dependent activating kinase; CCND1: cyclin D1; CCNH: Cyclin H; CDKN1A: cyclin-dependent kinase inhibitor 1A; CDKN1B: cyclin-dependent kinase inhibitor 1B; CHG: chromogranin; CR: castrationrecurrent; CRPC: castration-recurrent prostate cancer; CUEDC2: CUE-domaincontaining-2; DHCR24: 24-dehydrocholesterol reductase; DHRS7: dehydrogenase/reductase SDR-family member 7; EASE: Expression Analysis Systematic Explorer; ELOVL5: elongation of long chain fatty acids family member 5; ENO2: neuronal enolase 2; FLNA: filamin A; GO: Gene Ontology; HES6: hairy and enhancer of split 6; HGNC: HUGO Gene Nomenclature Committee; HMGB2: high mobility group box 2; HMGCS1: 3-hydroxy-3methylglutaryl-Coenzyme A synthase 1; HPA: hypothalamus-pituitary-adrenal; 
HSD17B3: hydroxysteroid (17-beta) dehydrogenase 3; HSD17B4: hydroxysteroid (17-beta) dehydrogenase 4; HSD17B5: hydroxysteroid (17beta) dehydrogenase 5; IL6: interleukin 6; KEGG: Kyoto Encyclopedia of Genes and Genomes; KLK3: kallikrein 3; KPNB1: karyopherin/importin beta 1; LHRH: Leutinizing hormone releasing hormone; LongSAGE: long serial analysis of gene expression; MAOA: monoamine oxidase A; NCOA: nuclear receptor coactivator; NKX3-1: NK3 homeobox 1; NTS: neurotensin; OPRK1: opioid receptor kappa 1; PKA: protein kinase A; PSA: prostate-specific antigen also known as KLK3; PSMA7: proteasome macropain subunit alpha type 7; PTHrP: parathyroid hormone-related protein; qRT-PCR: quantitative real timepolymerase chain reaction; RAD: responsive to androgen-deprivation; RAMP1: receptor (calicitonin) activity modifying protein 1; RB1: retinoblastoma 1; S100A10: S100 calcium binding protein A10; SQLE: squalene epoxidase; TRPM8: transient receptor potential cation channel subfamily $M$ member 8 .

\section{Acknowledgements}

The authors would like to thank Jean Wang for her excellent technical assistance and Dr. Simon Haile for helpful discussions. This work was supported by funding from NIH, Grant CA105304 (M.D.S.)

\section{Authors' contributions}

TLR and MDS conceived, designed, conducted, and analyzed all experiments described in this manuscript. TLR and MDS wrote the manuscript. GW performed the principle component analysis. MAM was responsible for SAGE library construction and sequencing. OM (tag clustering) and AD (library clustering) aided in bioinformatic analysis. All authors read and approved the final manuscript.

\section{Author's information}

M.D.S. and M.A.M. are Terry Fox Young Investigators. M.A.M. is a Senior Scholar of the Michael Smith Foundation for Health Research.

\section{Competing interests}

The authors declare that they have no competing interests.

Received: 20 April 2010 Accepted: 24 September 2010 Published: 24 September 2010

\section{References}

1. Feldman BJ, Feldman D: The development of androgen-independent prostate cancer. Nat Rev Cancer 2001, 1(1):34-45.

2. Scher HI, Sawyers $\mathrm{CL}$ : Biology of progressive, castration-resistant prostate cancer: directed therapies targeting the androgen-receptor signaling axis. J Clin Oncol 2005, 23(32):8253-8261.

3. So A, Gleave M, Hurtado-Col A, Nelson C: Mechanisms of the development of androgen independence in prostate cancer. World J Urol 2005, 23(1):1-9.

4. McPhaul MJ: Mechanisms of prostate cancer progression to androgen independence. Best Pract Res Clin Endocrinol Metab 2008, 22(2):373-388.

5. Visakorpi T, Hyytinen E, Koivisto P, Tanner M, Keinanen R, Palmberg C, Palotie A, Tammela T, Isola J, Kallioniemi OP: In vivo amplification of the androgen receptor gene and progression of human prostate cancer. Nat Genet 1995, 9(4):401-406.

6. Ford $\mathrm{OH}$, Gregory CW, Kim D, Smitherman AB, Mohler JL: Androgen receptor gene amplification and protein expression in recurrent prostate cancer. J Urol 2003, 170(5):1817-1821.

7. Gregory CW, He B, Johnson RT, Ford OH, Mohler JL, French FS, Wilson EM: A mechanism for androgen receptor-mediated prostate cancer recurrence after androgen deprivation therapy. Cancer Res 2001, 61(11):4315-4319.

8. Chmelar R, Buchanan G, Need EF, Tilley W, Greenberg NM: Androgen receptor coregulators and their involvement in the development and progression of prostate cancer. Int J Cancer 2007, 120(4):719-733.

9. Holzbeierlein J, Lal P, LaTulippe E, Smith A, Satagopan J, Zhang L, Ryan C, Smith S, Scher H, Scardino P, et al: Gene expression analysis of human prostate carcinoma during hormonal therapy identifies androgenresponsive genes and mechanisms of therapy resistance. Am J Pathol 2004, 164(1):217-227

10. Mostaghel EA, Nelson PS: Intracrine androgen metabolism in prostate cancer progression: mechanisms of castration resistance and therapeutic implications. Best Pract Res Clin Endocrinol Metab 2008, 22(2):243-258.
11. Labrie F: Adrenal androgens and intracrinology. Semin Reprod Med 2004, 22(4):299-309.

12. Veldscholte J, Berrevoets CA, Ris-Stalpers C, Kuiper GG, Jenster G, Trapman J, Brinkmann AO, Mulder E: The androgen receptor in LNCaP cells contains a mutation in the ligand binding domain which affects steroid binding characteristics and response to antiandrogens. J Steroid Biochem Mol Biol 1992, 41(3-8):665-669.

13. Culig Z, Hobisch A, Cronauer MV, Radmayr C, Trapman J, Hittmair A, Bartsch G, Klocker $\mathrm{H}$ : Androgen receptor activation in prostatic tumor cell lines by insulin-like growth factor-l, keratinocyte growth factor, and epidermal growth factor. Cancer Res 1994, 54(20):5474-5478.

14. Hobisch A, Eder IE, Putz T, Horninger W, Bartsch G, Klocker H, Culig Z: Interleukin- 6 regulates prostate-specific protein expression in prostate carcinoma cells by activation of the androgen receptor. Cancer Res 1998, 58(20):4640-4645.

15. Nazareth LV, Weigel NL: Activation of the human androgen receptor through a protein kinase A signaling pathway. J Biol Chem 1996, 271(33):19900-19907

16. Riegman PH, Vlietstra RJ, van der Korput JA, Brinkmann AO, Trapman J: The promoter of the prostate-specific antigen gene contains a functional androgen responsive element. Mol Endocrinol 1991, 5(12):1921-1930.

17. Yuan TC, Veeramani S, Lin MF: Neuroendocrine-like prostate cancer cells: neuroendocrine transdifferentiation of prostate adenocarcinoma cells. Endocr Relat Cancer 2007, 14(3):531-547.

18. Isaacs JT: The biology of hormone refractory prostate cancer. Why does it develop? Urol Clin North Am 1999, 26(2):263-273.

19. Velculescu VE, Zhang $L$, Vogelstein $B$, Kinzler KW: Serial analysis of gene expression. Science 1995, 270(5235):484-487.

20. Saha S, Sparks AB, Rago C, Akmaev V, Wang CJ, Vogelstein B, Kinzler KW, Velculescu VE: Using the transcriptome to annotate the genome. Nat Biotechnol 2002, 20(5):508-512.

21. Sadar MD, Akopian VA, Beraldi E: Characterization of a new in vivo hollow fiber model for the study of progression of prostate cancer to androgen independence. Mol Cancer Ther 2002, 1(8):629-637.

22. Wang G, Jones SJ, Marra MA, Sadar MD: Identification of genes targeted by the androgen and PKA signaling pathways in prostate cancer cells. Oncogene 2006, 25(55):7311-7323.

23. Wang G, Wang J, Sadar MD: Crosstalk between the androgen receptor and beta-catenin in castrate-resistant prostate cancer. Cancer Res 2008, 68(23):9918-9927.

24. Siddiqui AS, Khattra J, Delaney AD, Zhao Y, Astell C, Asano J, Babakaiff R, Barber S, Beland J, Bohacec S, et al: A mouse atlas of gene expression: large-scale digital gene-expression profiles from precisely defined developing C57BL/6J mouse tissues and cells. Proc Natl Acad Sci USA 2005, 102(51):18485-18490.

25. Gene Expression Omnibus. [http://www.ncbi.n/m.nih.gov/geo/].

26. DiscoverySpace. [http://www.bcgsc.ca/bioinfo/software/discoveryspace/]

27. Fitch WM, Margoliash E: Construction of phylogenetic trees. Science 1967, 155(760):279-284.

28. Felsenstein J: Numerical methods for inferring evolutionary trees. $Q$ Rev Biol 1982, 57:379-404.

29. Page RD: TreeView: an application to display phylogenetic trees on personal computers. Comput Appl Biosci 1996, 12(4):357-358.

30. Cai L, Huang H, Blackshaw S, Liu JS, Cepko C, Wong WH: Clustering analysis of SAGE data using a Poisson approach. Genome Biol 2004, 5(7):R51.

31. Blackshaw S, Harpavat S, Trimarchi J, Cai L, Huang H, Kuo WP, Weber G, Lee K, Fraioli RE, Cho SH, et al: Genomic analysis of mouse retinal development. PLOS Biol 2004, 2(9):E247.

32. Pruitt KD, Tatusova T, Maglott DR: NCBI reference sequences (RefSeq): a curated nonredundant sequence database of genomes, transcripts and proteins. Nucleic Acids Res 2007, , 35 Database: D61-65.

33. Robertson N, Oveisi-Fordorei M, Zuyderduyn SD, Varhol RJ, Fjell C, Marra M, Jones S, Siddiqui A: DiscoverySpace: an interactive data analysis application. Genome Biol 2007, 8(1):R6.

34. Ashburner M, Ball CA, Blake JA, Botstein D, Butler H, Cherry JM, Davis AP, Dolinski K, Dwight SS, Eppig JT, et al: Gene ontology: tool for the unification of biology. The Gene Ontology Consortium. Nat Genet 2000, 25(1):25-29.

35. Hosack DA, Dennis G Jr, Sherman BT, Lane HC, Lempicki RA: Identifying biological themes within lists of genes with EASE. Genome Biol 2003, 4(10):R70. 
36. Audic S, Claverie JM: The significance of digital gene expression profiles. Genome Res 1997, 7(10):986-995.

37. Kanehisa M, Araki M, Goto S, Hattori M, Hirakawa M, Itoh M, Katayama T, Kawashima S, Okuda S, Tokimatsu T, et al: KEGG for linking genomes to life and the environment. Nucleic Acids Res 2008, , 36 Database: D480-484.

38. Bairoch A, Apweiler R: The SWISS-PROT protein sequence database and its supplement TrEMBL in 2000. Nucleic Acids Res 2000, 28(1):45-48,

39. Al-Shahrour F, Minguez P, Tarraga J, Medina I, Alloza E, Montaner D, Dopazo J: FatiGO +: a functional profiling tool for genomic data. Integration of functional annotation, regulatory motifs and interaction data with microarray experiments. Nucleic Acids Res 2007, , 35 Web Server: W91-96

40. Strausberg RL, Feingold EA, Grouse LH, Derge JG, Klausner RD, Collins FS, Wagner L, Shenmen CM, Schuler GD, Altschul SF, et al: Generation and initial analysis of more than 15,000 full-length human and mouse CDNA sequences. Proc Natl Acad Sci USA 2002, 99(26):16899-16903.

41. Hubbard TJ, Aken BL, Beal K, Ballester B, Caccamo M, Chen Y, Clarke L, Coates G, Cunningham F, Cutts T, et al: Ensembl 2007. Nucleic Acids Res 2007, , 35 Database: D610-617.

42. Bismar TA, Demichelis F, Riva A, Kim R, Varambally S, He L, Kutok J, Aster JC, Tang J, Kuefer $R$, et al: Defining aggressive prostate cancer using a 12gene model. Neoplasia 2006, 8(1):59-68.

43. Shah RB, Mehra R, Chinnaiyan AM, Shen R, Ghosh D, Zhou M, Macvicar GR, Varambally S, Harwood J, Bismar TA, et al: Androgen-independent prostate cancer is a heterogeneous group of diseases: lessons from a rapid autopsy program. Cancer Res 2004, 64(24):9209-9216.

44. Wei Q, Li M, Fu X, Tang R, Na Y, Jiang M, Li Y: Global analysis of differentially expressed genes in androgen-independent prostate cancer. Prostate Cancer Prostatic Dis 2007, 10(2):167-174.

45. Assikis VJ, Do KA, Wen S, Wang X, Cho-Vega JH, Brisbay S, Lopez R, Logothetis CJ, Troncoso P, Papandreou CN, et al: Clinical and biomarker correlates of androgenindependent, locally aggressive prostate cancer with limited metastatic potential. Clin Cancer Res 2004, 10(20):6770-6778

46. Stanbrough M, Bubley GJ, Ross K, Golub TR, Rubin MA, Penning TM, Febbo PG, Balk SP: Increased expression of genes converting adrenal androgens to testosterone in androgenindependent prostate cancer. Cancer Res 2006, 66(5):2815-2825.

47. Best CJ, Gillespie JW, Yi Y, Chandramouli GV, Perlmutter MA, Gathright Y, Erickson HS, Georgevich L, Tangrea MA, Duray PH, et al: Molecular alterations in primary prostate cancer after androgen ablation therapy. Clin Cancer Res 2005, 11(19 Pt 1):6823-6834.

48. Tamura K, Furihata M, Tsunoda T, Ashida S, Takata R, Obara W, Yoshioka H, Daigo Y, Nasu Y, Kumon H, et al: Molecular features of hormonerefractory prostate cancer cells by genomewide gene expression profiles. Cancer Res 2007, 67(11):5117-5125.

49. Chandran UR, Ma C, Dhir R, Bisceglia M, Lyons-Weiler M, Liang W, Michalopoulos G, Becich M, Monzon FA: Gene expression profiles of prostate cancer reveal involvement of multiple molecular pathways in the metastatic process. BMC Cancer 2007, 7:64.

50. Zellweger T, Ninck C, Bloch M, Mirlacher M, Koivisto PA, Helin HJ, Mihatsch MJ, Gasser TC, Bubendorf L: Expression patterns of potential therapeutic targets in prostate cancer. Int J Cancer 2005, 113(4):619-628.

51. Fromont $G$, Chene $L$, Vidaud $M$, Vallancien $G$, Mangin P, Fournier $G$, Validire P, Latil A, Cussenot O: Differential expression of 37 selected genes in hormone-refractory prostate cancer using quantitative taqman realtime RT-PCR. Int J Cancer 2005, 114(2):174-181.

52. Bibikova M, Chudin E, Arsanjani A, Zhou L, Garcia EW, Modder J, Kostelec M, Barker D, Downs T, Fan JB, et al: Expression signatures that correlated with Gleason score and relapse in prostate cancer. Genomics 2007, 89(6):666-672.

53. Kumar-Sinha C, Chinnaiyan AM: Molecular markers to identify patients at risk for recurrence after primary treatment for prostate cancer. Urology 2003, 62(Suppl 1):19-35.

54. Stephenson AJ, Smith A, Kattan MW, Satagopan J, Reuter VE, Scardino PT, Gerald WL: Integration of gene expression profiling and clinical variables to predict prostate carcinoma recurrence after radical prostatectomy. Cancer 2005, 104(2):290-298.

55. Henshall SM, Afar DE, Hiller J, Horvath LG, Quinn DI, Rasiah KK, Gish K, Willhite $D$, Kench JG, Gardiner-Garden M, et al: Survival analysis of genome-wide gene expression profiles of prostate cancers identifies new prognostic targets of disease relapse. Cancer Res 2003, 63(14):4196-4203.

56. Glinsky GV, Glinskii AB, Stephenson AJ, Hoffman RM, Gerald WL: Gene expression profiling predicts clinical outcome of prostate cancer. J Clin Invest 2004, 113(6):913-923.

57. Febbo PG, Sellers WR: Use of expression analysis to predict outcome after radical prostatectomy. J Urol 2003, 170(6 Pt 2):S11-19, discussion S19-20.

58. Lapointe J, Li C, Higgins JP, van de Rijn M, Bair E, Montgomery K, Ferrari M, Egevad L, Rayford W, Bergerheim U, et al: Gene expression profiling identifies clinically relevant subtypes of prostate cancer. Proc Natl Acad Sci USA 2004, 101(3):811-816.

59. Devilard E, Bladou F, Ramuz O, Karsenty G, Dales JP, Gravis G, Nguyen C, Bertucci $F$, Xerri L, Birnbaum D: FGFR1 and WT1 are markers of human prostate cancer progression. BMC Cancer 2006, 6:272.

60. Quayle SN, Hare H, Delaney AD, Hirst M, Hwang D, Schein JE, Jones SJ, Marra MA, Sadar MD: Novel expressed sequences identified in a model of androgen independent prostate cancer. BMC Genomics 2007, 8:32.

61. Amler LC, Agus DB, LeDuc C, Sapinoso ML, Fox WD, Kern S, Lee D, Wang V Leysens M, Higgins B, et al: Dysregulated expression of androgenresponsive and nonresponsive genes in the androgen-independent prostate cancer xenograft model CWR22-R1. Cancer Res 2000, 60(21):6134-6141.

62. Chen Q, Watson JT, Marengo SR, Decker KS, Coleman I, Nelson PS, Sikes RA: Gene expression in the LNCaP human prostate cancer progression model: progression associated expression in vitro corresponds to expression changes associated with prostate cancer progression in vivo. Cancer Lett 2006, 244(2):274-288.

63. Morgenbesser SD, McLaren RP, Richards B, Zhang M, Akmaev VR, Winter SF, Mineva ND, Kaplan-Lefko PJ, Foster BA, Cook BP, et al: Identification of genes potentially involved in the acquisition of androgen-independent and metastatic tumor growth in an autochthonous genetically engineered mouse prostate cancer model. Prostate 2007, 67(1):83-106

64. Kuruma H, Egawa S, Oh-Ishi M, Kodera Y, Satoh M, Chen W, Okusa H, Matsumoto K, Maeda T, Baba S: High molecular mass proteome of androgen-independent prostate cancer. Proteomics 2005, 5(4):1097-1112.

65. Pfundt R, Smit F, Jansen C, Aalders T, Straatman H, van der Vliet W, Isaacs J, van Kessel AG, Schalken J: Identification of androgen-responsive genes that are alternatively regulated in androgen-dependent and androgenindependent rat prostate tumors. Genes Chromosomes Cancer 2005, 43(3):273-283.

66. Bubendorf L, Kolmer M, Kononen J, Koivisto P, Mousses S, Chen Y, Mahlamaki E, Schraml P, Moch $H$, Willi N, et al: Hormone therapy failure in human prostate cancer: analysis by complementary DNA and tissue microarrays. J Natl Cancer Inst 1999, 91(20):1758-1764.

67. Mousses $S$, Wagner U, Chen $Y$, Kim JW, Bubendorf $L$, Bittner M, Pretlow $T$, Elkahloun AG, Trepel JB, Kallioniemi OP: Failure of hormone therapy in prostate cancer involves systematic restoration of androgen responsive genes and activation of rapamycin sensitive signaling. Oncogene 2001, 20(46):6718-6723.

68. Gregory CW, Hamil KG, Kim D, Hall SH, Pretlow TG, Mohler JL, French FS: Androgen receptor expression in androgen-independent prostate cancer is associated with increased expression of androgen-regulated genes. Cancer Res 1998, 58(24):5718-5724.

69. Mohler UL, Morris TL, Ford OH, Alvey RF, Sakamoto C, Gregory CW: Identification of differentially expressed genes associated with androgen-independent growth of prostate cancer. Prostate 2002, 51(4):247-255.

70. Romanuik T, Wang G, Holt RA, Jones SJM, Marra MA, Sadar MD: Identification of novel androgen-responsive genes by sequencing of LongSAGE libraries. BMC Genomics 2009, 10(476).

71. Nelson PS, Clegg N, Arnold H, Ferguson C, Bonham M, White J, Hood L, Lin B: The program of androgen-responsive genes in neoplastic prostate epithelium. Proc Natl Acad Sci USA 2002, 99(18):11890-11895.

72. Oosterhoff JK, Grootegoed JA, Blok LJ: Expression profiling of androgendependent and - independent LNCaP cells: EGF versus androgen signalling. Endocr Relat Cancer 2005, 12(1):135-148.

73. Velasco AM, Gillis KA, Li Y, Brown EL, Sadler TM, Achilleos M, Greenberger LM, Frost P, Bai W, Zhang Y: Identification and validation of novel androgen-regulated genes in prostate cancer. Endocrinology 2004, 145(8):3913-3924. 
74. Segawa T, Nau ME, Xu LL, Chilukuri RN, Makarem M, Zhang W, Petrovics G, Sesterhenn IA, McLeod DG, Moul JW, et al: Androgen-induced expression of endoplasmic reticulum (ER) stress response genes in prostate cancer cells. Oncogene 2002, 21(57):8749-8758.

75. Xu LL, Su YP, Labiche R, Segawa T, Shanmugam N, McLeod DG, Moul JW, Srivastava S: Quantitative expression profile of androgen-regulated genes in prostate cancer cells and identification of prostate-specific genes. Int $J$ Cancer 2001, 92(3):322-328.

76. Clegg N, Eroglu B, Ferguson C, Arnold H, Moorman A, Nelson PS: Digital expression profiles of the prostate androgen-response program. J Steroid Biochem Mol Biol 2002, 80(1):13-23.

77. Coutinho-Camillo CM, Salaorni S, Sarkis AS, Nagai MA: Differentially expressed genes in the prostate cancer cell line LNCaP after exposure to androgen and anti-androgen. Cancer Genet Cytogenet 2006, 166(2):130-138.

78. DePrimo SE, Diehn M, Nelson JB, Reiter RE, Matese J, Fero M, Tibshirani R, Brown PO, Brooks JD: Transcriptional programs activated by exposure of human prostate cancer cells to androgen. Genome Biol 2002, 3(7): RESEARCH0032.

79. Febbo PG, Lowenberg M, Thorner AR, Brown M, Loda M, Golub TR: Androgen mediated regulation and functional implications of fkbp51 expression in prostate cancer. J Urol 2005, 173(5):1772-1777.

80. Meehan KL, Sadar MD: Quantitative profiling of LNCaP prostate cancer cells using isotopecoded affinity tags and mass spectrometry. Proteomics 2004, 4(4):1116-1134

81. Waghray A, Feroze F, Schober MS, Yao F, Wood C, Puravs E, Krause M, Hanash S, Chen YQ: Identification of androgen-regulated genes in the prostate cancer cell line LNCaP by serial analysis of gene expression and proteomic analysis. Proteomics 2001, 1(10):1327-1338.

82. Romanuik TL, Ueda T, Le N, Haile S, Yong TM, Thomson T, Vessella RL Sadar MD: Novel biomarkers for prostate cancer including noncoding transcripts. Am J Pathol 2009, 175(6):2264-2276.

83. Srikantan V, Zou Z, Petrovics G, Xu L, Augustus M, Davis L, Livezey JR, Connell T, Sesterhenn IA, Yoshino K, et al: PCGEM1, a prostate-specific gene, is overexpressed in prostate cancer. Proc Natl Acad Sci USA 2000, 97(22):12216-12221.

84. Hubert RS, Vivanco I, Chen E, Rastegar S, Leong K, Mitchell SC, Madraswala R, Zhou $Y$, Kuo J, Raitano AB, et al: STEAP: a prostate-specific cell-surface antigen highly expressed in human prostate tumors. ProC Natl Acad Sci USA 1999, 96(25):14523-14528.

85. Wang $R, X u$ J, Saramaki O, Visakorpi T, Sutherland WM, Zhou J, Sen B, Lim SD, Mabjeesh N, Amin M, et al: PrLZ, a novel prostate-specific and androgen-responsive gene of the TPD52 family, amplified in chromosome 8q21.1 and overexpressed in human prostate cancer. Cancer Res 2004, 64(5):1589-1594.

86. Waghray A, Schober M, Feroze F, Yao F, Virgin J, Chen YQ: Identification of differentially expressed genes by serial analysis of gene expression in human prostate cancer. Cancer Res 2001, 61(10):4283-4286.

87. Xu J, Stolk JA, Zhang X, Silva SJ, Houghton RL, Matsumura M, Vedvick TS, Leslie KB, Badaro R, Reed SG: Identification of differentially expressed genes in human prostate cancer using subtraction and microarray. Cancer Res 2000, 60(6):1677-1682.

88. Singh D, Febbo PG, Ross K, Jackson DG, Manola J, Ladd C, Tamayo P, Renshaw AA, D'Amico AV, Richie JP, et al: Gene expression correlates of clinical prostate cancer behavior. Cancer Cell 2002, 1(2):203-209.

89. Luo J, Duggan DJ, Chen Y, Sauvageot J, Ewing CM, Bittner ML, Trent JM, Isaacs WB: Human prostate cancer and benign prostatic hyperplasia: molecular dissection by gene expression profiling. Cancer Res 2001, 61(12):4683-4688.

90. Ernst T, Hergenhahn M, Kenzelmann M, Cohen CD, Bonrouhi M, Weninger A, Klaren R, Grone EF, Wiesel M, Gudemann C, et al: Decrease and gain of gene expression are equally discriminatory markers for prostate carcinoma: a gene expression analysis on total and microdissected prostate tissue. Am J Pathol 2002, 160(6):2169-2180.

91. Chaib H, Cockrell EK, Rubin MA, Macoska JA: Profiling and verification of gene expression patterns in normal and malignant human prostate tissues by cDNA microarray analysis. Neoplasia 2001, 3(1):43-52.

92. Ashida $S$, Nakagawa H, Katagiri T, Furihata M, liizumi M, Anazawa $Y$, Tsunoda T, Takata R, Kasahara K, Miki T, et al: Molecular features of the transition from prostatic intraepithelial neoplasia (PIN) to prostate cancer: genome-wide gene-expression profiles of prostate cancers and PINs. Cancer Res 2004, 64(17):5963-5972.
93. Rhodes DR, Barrette TR, Rubin MA, Ghosh D, Chinnaiyan AM: Meta-analysis of microarrays: interstudy validation of gene expression profiles reveals pathway dysregulation in prostate cancer. Cancer Res 2002, 62(15):4427-4433.

94. Latil A, Bieche I, Chene L, Laurendeau I, Berthon P, Cussenot O, Vidaud M: Gene expression profiling in clinically localized prostate cancer: a fourgene expression model predicts clinical behavior. Clin Cancer Res 2003, 9(15):5477-5485.

95. Li HR, Wang-Rodriguez J, Nair TM, Yeakley JM, Kwon YS, Bibikova M, Zheng C, Zhou L, Zhang K, Downs T, et al: Two-dimensional transcriptome profiling: identification of messenger RNA isoform signatures in prostate cancer from archived paraffin-embedded cancer specimens. Cancer Res 2006, 66(8):4079-4088.

96. Stamey TA, Warrington JA, Caldwell MC, Chen Z, Fan Z, Mahadevappa M, McNeal JE, Nolley R, Zhang Z: Molecular genetic profiling of Gleason grade $4 / 5$ prostate cancers compared to benign prostatic hyperplasia. J Urol 2001, 166(6):2171-2177.

97. Varambally S, Yu J, Laxman B, Rhodes DR, Mehra R, Tomlins SA, Shah RB, Chandran U, Monzon FA, Becich MJ, et al: Integrative genomic and proteomic analysis of prostate cancer reveals signatures of metastatic progression. Cancer Cell 2005, 8(5):393-406.

98. Chetcuti A, Margan S, Mann S, Russell P, Handelsman D, Rogers J, Dong Q: Identification of differentially expressed genes in organ-confined prostate cancer by gene expression array. Prostate 2001, 47(2):132-140.

99. Dhanasekaran SM, Barrette TR, Ghosh D, Shah R, Varambally S, Kurachi K, Pienta KJ, Rubin MA, Chinnaiyan AM: Delineation of prognostic biomarkers in prostate cancer. Nature 2001, 412(6849):822-826.

100. Magee JA, Araki T, Patil S, Ehrig T, True L, Humphrey PA, Catalona WJ, Watson MA, Milbrandt J: Expression profiling reveals hepsin overexpression in prostate cancer. Cancer Res 2001, 61(15):5692-5696.

101. Bull JH, Ellison G, Patel A, Muir G, Walker M, Underwood M, Khan F, Paskins L: Identification of potential diagnostic markers of prostate cancer and prostatic intraepithelial neoplasia using CDNA microarray. $\mathrm{Br}$ J Cancer 2001, 84(11):1512-1519.

102. Luo JH, Yu YP, Cieply K, Lin F, Deflavia P, Dhir R, Finkelstein S, Michalopoulos G, Becich M: Gene expression analysis of prostate cancers. Mol Carcinog 2002, 33(1):25-35.

103. Gleason DF, Mellinger GT: Prediction of prognosis for prostatic adenocarcinoma by combined histological grading and clinical staging. J Urol 1974, 111(1):58-64.

104. True L, Coleman I, Hawley S, Huang CY, Gifford D, Coleman R, Beer TM, Gelmann E, Datta M, Mostaghel E, et al: A molecular correlate to the Gleason grading system for prostate adenocarcinoma. Proc Natl Acad Sci USA 2006, 103(29):10991-10996.

105. LaTulippe E, Satagopan J, Smith A, Scher H, Scardino P, Reuter V, Gerald WL: Comprehensive gene expression analysis of prostate cancer reveals distinct transcriptional programs associated with metastatic disease. Cancer Res 2002, 62(15):4499-4506.

106. Ramaswamy S, Ross KN, Lander ES, Golub TR: A molecular signature of metastasis in primary solid tumors. Nat Genet 2003, 33(1):49-54.

107. Lee DK, Duan $\mathrm{HO}$, Chang C: From androgen receptor to the general transcription factor TFIIH. Identification of cdk activating kinase (CAK) as an androgen receptor $\mathrm{NH}(2)$-terminal associated coactivator. J Biol Chem 2000, 275(13):9308-9313.

108. Lin HK, Altuwaijri S, Lin WJ, Kan PY, Collins LL, Chang C: Proteasome activity is required for androgen receptor transcriptional activity via regulation of androgen receptor nuclear translocation and interaction with coregulators in prostate cancer cells. J Biol Chem 2002, 277(39):36570-36576.

109. Zhang PJ, Zhao J, Li HY, Man JH, He K, Zhou T, Pan X, Li AL, Gong WL, Jin $B F$, et al: CUE domain containing 2 regulates degradation of progesterone receptor by ubiquitinproteasome. Embo J 2007, 26(7):1831-1842

110. Loy CJ, Sim KS, Yong EL: Filamin-A fragment localizes to the nucleus to regulate androgen receptor and coactivator functions. Proc Natl Acad SCi USA 2003, 100(8):4562-4567.

111. Boonyaratanakornkit $V$, Melvin $V$, Prendergast $P$, Altmann $M$, Ronfani $L$, Bianchi ME, Taraseviciene L, Nordeen SK, Allegretto EA, Edwards DP: Highmobility group chromatin proteins 1 and 2 functionally interact with steroid hormone receptors to enhance their DNA binding in vitro and 
transcriptional activity in mammalian cells. Mol Cell Biol 1998, 18(8):4471-4487.

112. Mohler JL, Gregory CW, Ford OH, Kim D, Weaver CM, Petrusz P, Wilson EM, French FS: The androgen axis in recurrent prostate cancer. Clin Cancer Res 2004, 10(2):440-448.

113. Waterham HR, Koster J, Romeijn GJ, Hennekam RC, Vreken P, Andersson HC, FitzPatrick DR, Kelley RI, Wanders RJ: Mutations in the 3beta-hydroxysterol Delta24-reductase gene cause desmosterolosis, an autosomal recessive disorder of cholesterol biosynthesis. Am J Hum Genet 2001, 69(4):685-694.

114. Haeseleer F, Palczewski K: Short-chain dehydrogenases/reductases in retina. Methods Enzymol 2000, 316:372-383.

115. Momozawa Y, Takeuchi Y, Kitago M, Masuda K, Kakuma Y, Hashizume C, Ichimaru T, Mogi K, Okamura H, Yonezawa T, et al: Gene expression profiles linked to the hormonal induction of male-effect pheromone synthesis in goats (Capra hircus). Biol Reprod 2007, 77(1):102-107.

116. Kitago M, Momozawa Y, Masuda K, Wakabayashi Y, Date-lto A, HaginoYamagishi K, Kikusui T, Takeuchi Y, Mori Y: Localization of the candidate genes ELOVL5 and SCD1 for 'male effect' pheromone synthesis in goats (Capra hircus). J Reprod Dev 2007, 53(6):1329-1333.

117. Penning TM: Hydroxysteroid dehydrogenases and pre-receptor regulation of steroid hormone action. Hum Reprod Update 2003 9(3):193-205.

118. Pascoe JE, Williams KL, Mukhopadhyay P, Rice KC, Woods JH, Ko MC: Effects of mu, kappa, and delta opioid receptor agonists on the function of hypothalamic-pituitary-adrenal axis in monkeys. Psychoneuroendocrinology 2008, 33(4):478-486.

119. Hu Y, Wang T, Stormo GD, Gordon Jl: RNA interference of achaete-scute homolog 1 in mouse prostate neuroendocrine cells reveals its gene targets and DNA binding sites. Proc Natl Acad Sci USA 2004, 101(15):5559-5564.

120. Lam MH, Thomas RJ, Loveland KL, Schilders S, Gu M, Martin TJ, Gillespie MT, Jans DA: Nuclear transport of parathyroid hormone (PTH)-related protein is dependent on microtubules. Mol Endocrinol 2002, 16(2):390-401.

121. Zhao H, Nolley R, Chen Z, Reese SW, Peehl DM: Inhibition of monoamine oxidase A promotes secretory differentiation in basal prostatic epithelial cells. Differentiation 2008, 76(7):820-830.

122. Abrahamsson PA: Neuroendocrine differentiation in prostatic carcinoma. Prostate 1999, 39(2):135-148.

123. Svenningsson $P$, Chergui $K$, Rachleff I, Flajolet $M$, Zhang $X$, El Yacoubi M, Vaugeois JM, Nomikos GG, Greengard P: Alterations in 5-HT1B receptor function by $\mathrm{p} 11$ in depressionlike states. Science 2006, 311(5757):77-80.

124. Mergler S, Strowski MZ, Kaiser S, Plath T, Giesecke Y, Neumann M, Hosokawa H, Kobayashi S, Langrehr J, Neuhaus $P$, et al: Transient receptor potential channel TRPM 8 agonists stimulate calcium influx and neurotensin secretion in neuroendocrine tumor cells. Neuroendocrinology 2007, 85(2):81-92.

125. Mourtada-Maarabouni M, Hedge VL, Kirkham L, Farzaneh F, Williams GT: Growth arrest in human T-cells is controlled by the non-coding RNA growth-arrest-specific transcript 5 (GAS5). J Cell Sci 2008, 121(Pt 7):939-946.

126. Hermanto $U$, Zong CS, Li W, Wang LH: RACK1, an insulin-like growth factor I (IGF-I) receptor-interacting protein, modulates IGF-I-dependent integrin signaling and promotes cell spreading and contact with extracellular matrix. Mol Cell Biol 2002, 22(7):2345-2365.

127. Kim MJ, Bhatia-Gaur R, Banach-Petrosky WA, Desai N, Wang Y, Hayward SW, Cunha GR, Cardiff RD, Shen MM, Abate-Shen C: Nkx3.1 mutant mice recapitulate early stages of prostate carcinogenesis. Cancer Res 2002, 62(11):2999-3004.

128. Petrovics G, Zhang W, Makarem M, Street JP, Connelly R, Sun L, Sesterhenn IA, Srikantan V, Moul JW, Srivastava S: Elevated expression of PCGEM1, a prostate-specific gene with cell growth-promoting function, is associated with high-risk prostate cancer patients. Oncogene 2004, 23(2):605-611.

129. Wang S, Yang Q, Fung KM, Lin HK: AKR1C2 and AKR1C3 mediated prostaglandin $\mathrm{D}(2)$ metabolism augments the PI3K/Akt proliferative signaling pathway in human prostate cancer cells. Mol Cell Endocrinol 2008, 289(1-2):60-66.

130. Challita-Eid PM, Morrison K, Etessami S, An Z, Morrison KJ, Perez-Villar JJ, Raitano AB, Jia XC, Gudas JM, Kanner SB, et al: Monoclonal antibodies to six-transmembrane epithelial antigen of the prostate-1 inhibit intercellular communication in vitro and growth of human tumor xenografts in vivo. Cancer Res 2007, 67(12):5798-5805.

131. Katoh Y, Katoh M: Identification and characterization of CDC50A, CDC50B and CDC50C genes in silico. Oncol Rep 2004, 12(4):939-943.

132. Matsuda S, Rouault J, Magaud J, Berthet C: In search of a function for the TIS21/PC3/BTG1/TOB family. FEBS Lett 2001, 497(2-3):67-72.

133. Trueb B, Zhuang $L$, Taeschler S, Wiedemann M: Characterization of FGFRL1, a novel fibroblast growth factor (FGF) receptor preferentially expressed in skeletal tissues. J Biol Chem 2003, 278(36):33857-33865.

134. Anazawa Y, Nakagawa H, Furihara M, Ashida S, Tamura K, Yoshioka H, Shuin T, Fujioka T, Katagiri T, Nakamura Y: PCOTH, a novel gene overexpressed in prostate cancers, promotes prostate cancer cell growth through phosphorylation of oncoprotein TAF-lbeta/SET. Cancer Res 2005, 65(11):4578-4586.

135. Sakamoto H, Mashima T, Kizaki A, Dan S, Hashimoto Y, Naito M, Tsuruo T: Glyoxalase I is involved in resistance of human leukemia cells to antitumor agent-induced apoptosis. Blood 2000, 95(10):3214-3218.

136. Hsu SY, Kaipia A, Zhu L, Hsueh AJ: Interference of BAD (Bcl-xL/Bcl-2associated death promoter)-induced apoptosis in mammalian cells by 14-3-3 isoforms and P11. Mol Endocrinol 1997, 11(12):1858-1867.

137. Zhang L, Barritt GJ: Evidence that TRPM 8 is an androgen-dependent Ca2 + channel required for the survival of prostate cancer cells. Cancer Res 2004, 64(22):8365-8373

138. Lin J, Adam RM, Santiestevan E, Freeman MR: The phosphatidylinositol 3'kinase pathway is a dominant growth factor-activated cell survival pathway in LNCaP human prostate carcinoma cells. Cancer Res 1999, 59(12):2891-2897.

139. Rokhlin OW, Taghiyev AF, Bayer KU, Bumcrot D, Koteliansk VE, Glover RA, Cohen MB: Calcium/calmodulin-dependent kinase II plays an important role in prostate cancer cell survival. Cancer Biol Ther 2007, 6(5):732-742.

140. Spiess C, Meyer AS, Reissmann S, Frydman J: Mechanism of the eukaryotic chaperonin: protein folding in the chamber of secrets. Trends Cell Biol 2004, 14(11):598-604.

141. Kadomatsu K, Muramatsu T: Midkine and pleiotrophin in neural development and cancer. Cancer Lett 2004, 204(2):127-143.

142. You Z, Dong Y, Kong X, Beckett LA, Gandour-Edwards R, Melamed J: Midkine is a NFkappaB-inducible gene that supports prostate cancer cell survival. BMC Med Genomics 2008, 1:6

143. Mannherz O, Mertens D, Hahn M, Lichter P: Functional screening for proapoptotic genes by reverse transfection cell array technology. Genomics 2006, 87(5):665-672.

144. Mellor HR, Harris AL: The role of the hypoxia-inducible $\mathrm{BH} 3$-only proteins BNIP3 and BNIP3L in cancer. Cancer Metastasis Rev 2007, 26(3-4):553-566.

145. Proikas-Cezanne T, Waddell S, Gaugel A, Frickey T, Lupas A, Nordheim A: WIPI-1alpha (WIPI49), a member of the novel 7-bladed WIPI protein family, is aberrantly expressed in human cancer and is linked to starvation-induced autophagy. Oncogene 2004, 23(58):9314-9325.

146. Mukai J, Hachiya T, Shoji-Hoshino S, Kimura MT, Nadano D, Suvanto P, Hanaoka T, Li Y, Irie S, Greene LA, et al: NADE, a p75NTR-associated cell death executor, is involved in signal transduction mediated by the common neurotrophin receptor p75NTR. J Biol Chem 2000, 275(23):17566-17570.

147. Krygier S, Djakiew D: Molecular characterization of the loss of p75(NTR) expression in human prostate tumor cells. Mol Carcinog 2001, 31(1):46-55.

\section{Pre-publication history}

The pre-publication history for this paper can be accessed here: http://www.biomedcentral.com/1755-8794/3/43/prepub

doi:10.1186/1755-8794-3-43

Cite this article as: Romanuik et al.: LNCaP Atlas: Gene expression associated with in vivo progression to castration-recurrent prostate cancer. BMC Medical Genomics 2010 3:43. 